$7 \quad{ }^{1}$ Basic Sciences Division and ${ }^{3}$ Howard Hughes Medical Institute, Fred Hutchinson Cancer

8 Research Center, Seattle WA

$9 \quad{ }^{2}$ Department of Physiology and Biophysics, University of Washington, Seattle WA

10 *Co-corresponding authors: sbiggins@fredhutch.org; casbury@uw.edu 


\section{Introduction / Abstract}

Chromosome segregation requires large macromolecular structures called kinetochores to attach dynamic microtubules from opposite spindle poles ${ }^{1,2}$. Attachments are made iteratively, through a trial-and-error process, and proper attachments come under tension from the pulling forces of microtubules ${ }^{3,4}$. However, if sister kinetochores bind microtubules from the same pole ${ }^{1,2}$, these defective attachments lack tension and must be destabilized to give another chance for proper attachments to form. This vital error correction process requires Aurora B kinase, which phosphorylates kinetochores lacking tension to reduce their affinity for microtubules ${ }^{5-11}$. An unresolved question is how Aurora B distinguishes the level of tension on kinetochores. There are conflicting reports on the underlying mechanism ${ }^{12-16}$, owing in part to the difficulties of manipulating kinetochore tension in vivo and distinguishing kinase from opposing phosphatase activity. To address these issues, we have reconstituted Aurora B-triggered kinetochore detachment in an in vitro optical trapping-based flow assay. Here, we test an outstanding model by determining whether kinetochore tension is sufficient to prevent kinase-triggered detachments. Strikingly, Aurora B detaches kinetochores from microtubules under both high and low tension, providing direct evidence that the kinase does not distinguish correct versus incorrect attachments by recognizing tension-dependent changes in the conformation of its kinetochore substrates.

\section{Main}

Aurora $\mathrm{B}$ is a conserved and essential mitotic kinase that ensures accurate chromosome segregation and has been implicated in oncogenesis ${ }^{17-19}$. It is the catalytic component of the four-

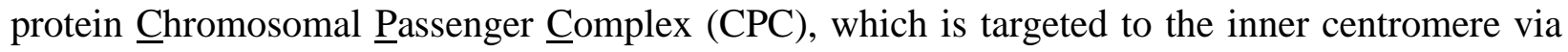
the Survivin and Borealin proteins ${ }^{20,21}$. The fourth member of the complex, INCENP, acts as a 
34 scaffold and activates the kinase through its C-terminus ${ }^{22-27}$ (Fig. 1a). Aurora B is additionally 35 activated by autophosphorylation in trans, and it is thought that clustering at the inner centromere facilitates this autoactivation before a centromere-centered diffusion gradient enables access to

37 distant outer-kinetochore substrates that contact microtubules ${ }^{28}$. Much work has been done to identify Aurora B phosphorylation sites on the microtubule-binding elements of the kinetochore.

When purified microtubule-binding kinetochore proteins are either pre-phosphorylated by Aurora B or engineered with phosphomimetic mutations at its target sites, they exhibit weakened affinity

41 for microtubules ${ }^{8,29,30}$.

A major outstanding question is how Aurora B distinguishes kinetochore-microtubule attachments lacking tension from those under tension. The observation that there is an Aurora B

44 activity gradient centered at the inner centromere led to an attractive model. According to this

45 "spatial separation" model, outer kinetochore substrates are accessible by inner centromere-

46 localized CPC only when a kinetochore lacks tension, but substrates become spatially distant from

47 the kinase once they come under tension ${ }^{12,13}$. This was the prevailing model before it was shown

48 that inner-centromeric localization of the CPC is dispensable for proper error correction in budding 49 yeast, chicken, and human cells ${ }^{14-16}$. An alternate "substrate conformation" model, which is

50 independent of kinase localization, proposes that conformational changes occurring when

51 kinetochores come under tension block Aurora B phosphorylation ${ }^{15,16,21,31}$. Electron microscopy,

52 fluorescence microscopy, and biochemical crosslinking studies have demonstrated that

53 kinetochores undergo large structural changes when they come under tension, which could 54 potentially block Aurora B phosphorylation via substrate occlusion or another mechanism ${ }^{32-34}$. 55 This model is analogous to the many known instances where tension-dependent conformational 
56 changes affect binding and regulation of protein complexes, such as with integrins ${ }^{35}$ or

57 mechanosensitive ion channels ${ }^{36}$.

It has not been possible to rigorously test models for Aurora B regulation within cells for

59 several reasons. Aurora B has additional mitotic roles, so kinase inhibition acts globally and not

60 specifically on error correction. In addition, it is challenging to test various models in vivo because

61 unattached kinetochores also lack tension, and kinetochores that make attachments quickly come

62 under tension. Finally, the phosphorylation state of individual kinetochores in vivo cannot be

63 precisely correlated with kinase activity because substrate phosphorylation represents a balance of

64 both kinase and opposing phosphatase activity ${ }^{37-40}$. These challenges led us to seek a controllable

65 in vitro system that mimics the turnover of kinetochore-microtubule attachments during

66 prometaphase and allows the regulation of Aurora B in this process to be studied more directly.

We previously developed an optical trap assay that reconstitutes kinetochore-microtubule

68 attachments under tension ${ }^{41}$. The kinetochores are purified from budding yeast and do not carry

69 Aurora $\mathrm{B}^{\mathrm{Ip} 11}$ because they lack some inner centromeric proteins. While we cannot test the spatial

70 separation model with these kinetochores, they provide an opportunity to test the substrate

71 conformation model by asking whether non-centromeric Aurora B can detach kinetochores from

72 microtubules under high tension. For such a test, we needed kinetochores lacking all kinase activity

73 to specifically monitor the activity of exogenous Aurora B. We previously found that purified

74 kinetochores isolated from wild-type strains contain active Mps1 kinase ${ }^{42}$, so we used kinetochores

75 from an mpsl-1 strain that had been shifted to the non-permissive temperature to inactivate Mps 1.

76 Consistent with previous findings in meiotic cells ${ }^{43}$, we found that Mps1-1 kinetochores lack co-

77 purifying Dam1 complex (Fig. S1), but otherwise resemble wild-type purified kinetochores ${ }^{42}$. To 
ensure there was no opposing phosphatase activity, all kinetochores were purified in the presence of irreversible phosphatase inhibitors.

We next needed a source of highly active Aurora B kinase to add to the kinetochores, so we constructed a chimeric construct that has catalytic activity but lacks the other members of the $\mathrm{CPC}$, including its centromeric targeting domains (Fig. 1a). Specifically, we fused the yeast INCENP $^{\text {Sli15 }}$ activation box (residues 624-698) to the N-terminus of yeast Aurora $\mathrm{B}^{\mathrm{Ip} 11}$ to create AurB*. We confirmed its activity by incubating the kinetochores with ATP- $\gamma-{ }^{32} \mathrm{P}$ and either AurB* or a kinase-dead mutant AurB* (AurB*-KD) where the catalytic lysine had been substituted with arginine (Fig. 1b). AurB* autoactivated and efficiently phosphorylated known kinetochore substrates Ndc80, Spc105 and Dsn1 13,44,45 (Fig. 1c). The key microtubule-coupling substrate Ndc80, on which a phospho-shift could be observed, was phosphorylated to greater than $50 \%$ completion in under one minute with $0.2 \mu \mathrm{M}$ AurB* (Fig. 1d). In contrast, there was minimal phosphorylation on kinetochores incubated with $0.2 \mu \mathrm{M}$ AurB*-KD, even after several minutes. No phosphorylation was observed on kinetochores that had been incubated only with ATP- $\gamma-{ }^{32} \mathrm{P}$, confirming the lack of endogenous kinase activity. To ensure that AurB* was phosphorylating physiological sites on the purified kinetochores, we repeated the experiment using phosphodeficient kinetochores with alanine substitutions at the seven known Aurora B sites on the Ndc80 N-terminus (Ndc80-7A $)^{44}$. Because the $n d c 80-7 A$ and $m p s 1-1$ mutations are synthetic lethal in yeast, we used kinetochores isolated from strains containing wild-type Mps1. Although we could then detect phosphorylation in the absence of AurB*, the phosphorylation of $\mathrm{Ndc} 80$ protein was clearly reduced in the phospho-deficient Ndc80-7A kinetochores when compared to wild-type, and the electrophoretic mobility of $\mathrm{Ndc} 80$ protein was increased (Fig. 1e). Thus, AurB* phosphorylates kinetochores on physiologically-relevant sites. 
When Aurora B is downregulated in cells, erroneous attachments accumulate a $^{5-4,46}$.

102

103

104

105

106

107

108

109

110

111

112

However, it has been difficult to establish the direct results of kinetochore phosphorylation by

Aurora B. Experiments in yeast suggest that chromosomes lacking tension detach from microtubules ${ }^{6}$, but native attachments cannot be directly visualized in these small cells. In large $\mathrm{PtK}_{2}$ cells where attachments can be seen, Aurora B inhibitor washout led to erroneously-attached kinetochores moving to cell poles instead of being immediately detached from microtubules ${ }^{7}$. We therefore asked whether Aurora B is sufficient to directly detach kinetochores from microtubules in vitro. We polymerized fluorescently-labeled tubulin into taxol-stabilized microtubules and incubated them with purified wild-type kinetochores that were retained on beads (Fig. 2a). Because the kinetochores lack stoichiometric amounts of the Dam1 complex, an important microtubulecoupling agent, we pre-incubated them with independently-purified native Dam1 complex ${ }^{41,47}$. The microtubule-bound kinetochores were then incubated with either buffer or AurB* and the quantity of bound microtubules was monitored over time. Incubating kinetochores with AurB* caused a phospho-shift in Ndc80 mobility and a decrease in bound microtubules over time, whereas both of these effects were absent in the kinetochores incubated with buffer alone (Figs. $2 \mathrm{~b}$ and $2 \mathrm{c}$ ). Incubation with AurB*-KD also did not cause microtubule release and prephosphorylation before microtubule incubation prevented microtubule binding (Figs. S2a and S2b). Previous studies suggest that AurB*-triggered microtubule release should primarily result from phosphorylation of microtubule-contacting residues on the Ndc80 and Dam1 proteins ${ }^{8,30,48,49}$. To confirm this, we repeated the experiment using phospho-deficient kinetochores purified from strains containing Ndc80-7A. We found that Ndc80-7A kinetochores released microtubules at a slower rate than wild-type kinetochores, but not as slowly as the no-kinase control (Figs. 2b and 2c), which confirms that these Ndc80 residues partially contributed to the release. Together, these 
124

125

126

127

128

129

130

observations provide direct evidence that Aurora B phosphorylation is sufficient for the release of kinetochore-microtubule attachments. Interestingly, the kinetochores in these experiments are likely making side-on attachments to the microtubule lattice ${ }^{41}$, a configuration similar to the initial attachments that form in prometaphase cells. Evidence suggests that side-on attachments in cells are immune to Aurora B error correction ${ }^{50}$. However, our results indicate that side-on-attached kinetochores are probably susceptible to Aurora-triggered release, suggesting that additional regulation might be required in vivo to protect them.

Kinetochores in cells make persistent, load-bearing attachments to dynamic microtubule tips and this arrangement is thought to be the relevant target for error correction. Therefore, we wanted to reconstitute tip-coupled kinetochores under differing amounts of tension to assay whether Aurora B phosphorylation could release these physiologically relevant attachments. We linked Mps 1-1 kinetochores to polystyrene microbeads and then used an optical trap to attach the beads individually to the assembling tips of single, coverslip-anchored microtubules, as in previous work $^{30,41,51-53}$. The rupture strength of tip-coupled Mps1-1 kinetochores was relatively low, presumably because they lack co-purifying Dam1 complex (Fig. S3a), so we again separately purified Dam1 complex and included it in the assay buffer. Tip-coupling strength was improved, and the Mps1-1 kinetochores could remain persistently attached to growing and shortening tips under a variety of tensile forces (Figs. S3a and S3b).

To test whether Aurora B could disrupt the attachments, we adapted the trapping assay to introduce AurB* into the reaction chamber by flowing in kinase-containing buffer once a tipcoupled kinetochore-microtubule attachment had been established (Fig. 3a). This approach was technically challenging. Unlike previous versions of our optical trap assay, only one kinetochore attachment could be probed per slide, because any free-floating unattached kinetochores became 
147 phosphorylated once AurB* was introduced and thus were unsuitable for further measurements.

148 Moreover, kinase introduction required flow in the chamber that caused extra beads to catch in the

149 laser, which ended the events prematurely (Fig. 3b, Movie 1). To minimize the frequency of such

150 interruptions, we kept the flowrate and the concentration of conjugated beads low, and we used

151 the weakest possible trap stiffness (see Materials \& Methods). ATP was included in the buffer

152 before and after the exchange. Under these modified assay conditions, the tip-tracking of

153 kinetochore beads was not detectably disturbed by the onset of flow (Figs. 3b and 3c).

To mimic erroneous attachments that should be corrected, we applied a constant low 155 tension of $\sim 1 \mathrm{pN}$ between a kinetochore-coupled bead and a microtubule ${ }^{41}$, introduced either 156 AurB $^{*}$ or AurB*-KD, and then monitored the bead until either detachment or interruption 157 (Fig. 4a). To avoid biasing the data towards higher detachment rates, we did not discard events 158 that were interrupted and instead calculated overall detachment rates by dividing the number of 159 detachments observed for a given condition by the total observation time after kinase flow was initiated. The spontaneous detachment rate in the presence of AurB*-KD was $2.3 \pm 0.9 \mathrm{hr}^{-1}$. When active AurB* was flowed in, the detachment rate was elevated 3 -fold to $7.2 \pm 2.2 \mathrm{hr}^{-1}$, 162 demonstrating that Aurora B can directly disrupt tip-coupled kinetochore-microtubule attachments 163 (Figs. 4b and 4c, Movie 2). These data demonstrate that Aurora B activity is sufficient to directly 164 detach kinetochores from microtubule tips in vitro.

We next sought to test one of the major models that has been proposed to explain the 166 tension-dependent suppression of Aurora B activity: the substrate conformation model. To 167 determine whether tension induces conformational changes that prevent Aurora B-mediated 168 detachments, we put kinetochores under high tension, applying a constant force of $5 \mathrm{pN}^{41}$, and 169 then flowed in either AurB* or AurB*-KD. The substrate conformation model predicts that 
detachment rates should be similarly low, with either AurB* or AurB*-KD, when the attachments are held under high tension. However, high force did not prevent Aurora B-mediated detachments and AurB* was still able to robustly trigger detachments at high force (Fig. 5a-c). One explanation for these results would be that we had not applied enough tension, so we collected data up to $8 \mathrm{pN}$, where detachment rates were high even in the absence of kinase activity. However, there was not observable suppression of AurB* activity at any force (Fig. S4a). We note that while previous experiments in the absence of kinase activity have shown that tension can suppress kinetochore detachment $^{41,53}$, we did not see this effect under the conditions used here, even in the presence of only AurB*-KD (Supplementary Note). To ensure that the elevated detachment rates we observed in the presence of active AurB* were due to kinase activity, we measured detachment rates at two different concentrations of AurB*: $5 \mu \mathrm{M}$ and $0.5 \mu \mathrm{M}$. At a high force where the effects of AurB* versus AurB*-KD flow were very different, detachment rates showed a clear dependence on enzyme concentration (Fig. 5b-c). These results were not artifacts of our mutant Mps1-1 kinetochores or the addition of Dam1 complex, as we also measured detachment rates of wild-type kinetochores from microtubules and found that AurB* again stimulated detachments (Fig. S4b). Together, these data strongly suggest that tension alone does not alter the ability of Aurora B to access its kinetochore substrates.

A number of models have been proposed to explain how Aurora B selectively weakens kinetochore-microtubule attachments that lack tension, while leaving load-bearing, tip-coupled kinetochores unmodified. Rigorously testing such models requires independent control of tension, attachment, and enzyme activity. Here, we developed an assay that fulfills these needs and allows components to be introduced while kinetochore-microtubule attachments are held continuously under tension. While previous work has shown that pre-phosphorylation reduces the microtubule 
193 binding affinity of individual kinetochore components ${ }^{8,29,30}$, our results provide the first direct

194 demonstration that Aurora B activity is sufficient to detach kinetochores from dynamic

195 microtubule tips. Recent work suggests that Aurora B's inner centromere localization is

196 dispensable for error correction in cells ${ }^{14-16}$, leading to the idea that changes in substrate

197 conformation might be responsible for its tension-dependent activity ${ }^{15,16,21,31}$. However, we find

198 that the kinase readily releases load-bearing kinetochore-microtubule attachments, indicating that

199 its activity is not suppressed by tension-induced conformational changes in its kinetochore

200 substrates. Several other mechanisms could explain the tension dependence of Aurora B, including

201 the possibility that tension directly across Aurora B or the CPC could inactivate the kinase ${ }^{54}$, or

202 that the phosphatase, rather than the kinase, could be regulated by tension. Immunofluorescence

203 has revealed a secondary pool of active Aurora B, close to the outer kinetochore and distinct from

204 the inner-centromeric pool ${ }^{55}$, leading to the attractive possibility that this secondary pool of

205 Aurora B might localize specifically to kinetochores lacking tension and be depleted only upon

206 the establishment of tension ${ }^{56}$. The flow assay we developed here can be used in the future to test

207 these other models of Aurora B-based error correction, to help uncover how this important kinase

208 senses tension and ultimately ensures the accuracy of chromosome segregation.

\section{Online Methods}

\section{Protein preparation}

The AurB* gene was created in several steps. First, the non-expressed linker between the

213 SLI15 and IPL1 genes was removed on two polycistronic vectors expressing the four members of

214 the CPC: pDD2396 (wild-type kinase) and pDD2399 (kinase-dead) ${ }^{57}$. A TEV cleavage site was

215 added between the 6xHis tag and the IPL1 gene in each plasmid and then the entire SLI15-IPL1- 
TEV-6xHis genes from these constructs were amplified via PCR with BamHI and XhoI sites on the 5' and 3' ends, respectively, and inserted them into cut pET21b vectors (EMD Biosciences). Each kinase was then expressed as a single polypeptide with a T7 tag on its N-terminus and a 219 6xHis tag on its C-terminus (plasmids pSB2540 and pSB2894 for wild-type and kinase-dead, respectively). For protein preparation, each plasmid was co-transformed into the Tuner pLys BL21 (DE3) E. coli strain (Novagen) with a plasmid that encodes lambda phosphatase ${ }^{58}$. A single transformant was grown to OD 0.9 and expression of both proteins induced with $500 \mu \mathrm{M}$ IPTG. Protein was expressed for four hours at $30{ }^{\circ} \mathrm{C}$ before harvesting. Cells were resuspended in prep buffer (50 mM Tris-HCl pH 7.4, 300 mM KCl, 5 mM MgCl 2,5 mM ATP, 1 mM DTT, 20 mM imidazole, $10 \%$ glycerol) supplemented with $1 \%$ Triton $\mathrm{X}-100,1 \mu \mathrm{L}$ Turbo nuclease (Accelagen) 226 and one tablet of Complete EDTA-free protease inhibitor (Roche) ${ }^{57}$ and then lysed via incubation 227 with $0.5 \mathrm{mg} / \mathrm{mL}$ lysozyme followed by sonication. Lysate was cleared by centrifugation and then 228 applied to a column of Ni-NTA resin (Qiagen). Column was washed with 10 column volumes 229 (CVs) of prep buffer and then protein-bound resin was resuspended in 3 CVs of prep buffer. DTT was added to $1 \mathrm{mM}$ and $3 \mathrm{mg}$ of TEV protease was added for three hours of room temperature 231 incubation. TEV protease was expressed and purified in house ${ }^{59}$. The resin slurry was re-applied to the empty column and the flow-through was collected along with the flow-through from one wash. This was concentrated and applied to a Superdex 200 FPLC column with storage buffer (50 $\mathrm{mM}$ Tris- $\mathrm{HCl} \mathrm{pH}$ 7.4, $300 \mathrm{mM} \mathrm{NaCl}, 10 \%$ glycerol, $50 \mathrm{mM}$ arginine, $50 \mathrm{mM}$ glutamate). Fractions were analyzed by SDS-PAGE and those from the main peak that eluted at $72 \mathrm{~mL}$ were pooled and concentrated. 
were purified from strain SBY8253; Mps1-1 kinetochores were from SBY8726; Ndc80-7A

kinetochores were from SBY8522. Yeast strains were grown asynchronously to OD 4 at room then left attached to beads. 


\section{Enzyme assays}

For the radioactive kinase assays shown in Fig. 1, kinetochores were purified and immobilized on magnetic beads and then incubated with AurB*, AurB*-KD, or buffer with 0.2 $\mu \mathrm{M}$ hot $\left({ }^{32} \mathrm{P}-\gamma\right.$-labeled) and $200 \mu \mathrm{M}$ cold ATP for the indicated periods of time at room

267 temperature. Experiments were quenched in sample buffer and separated by SDS-PAGE before

268 being silver stained. Gel was dried and exposed to a storage phosphor screen for longer than 48 269 hours for detection of incorporated ${ }^{32} \mathrm{P}$.

271 kinetochores were purified and immobilized on beads, incubated with $80 \mathrm{nM}$ purified Dam1 272 complex for 15 minutes, washed, then incubated in 1xBRB80 with $500 \mu \mathrm{M} \mathrm{ATP}, 1.8 \mathrm{mg} / \mathrm{mL} \kappa-$ 273 casein, $20 \mu \mathrm{M}$ taxol, and taxol-stabilized fluorescently-labeled microtubules for 5 minutes. At $\mathrm{t}=0$,

274 an aliquot was removed, washed with 1xBRB80 with $20 \mu \mathrm{M}$ taxol and $0.01 \%$ Tween-20, and 275 quenched with sample buffer. Buffer or $1 \mu \mathrm{M}$ AurB* was added to each experiment after initial 276 aliquot removal and further aliquots were removed, washed and quenched at the indicated times.

277 The entire experiment occurred at room temperature. Samples were eluted from beads via boiling, 278 separated by SDS-PAGE, and then analyzed via Western blot and fluorescence scan.

\section{Immunoblotting}

For Western blots, proteins were transferred from SDS-PAGE gels onto $0.22 \mu \mathrm{M}$ cellulose paper, blocked at room temperature with $4 \%$ milk in PBST, and incubated overnight at $4{ }^{\circ} \mathrm{C}$ in 
Millipore YL1/2 1:1000. Blots were then washed again with PBST and incubated with secondary antibody at room temperature. Secondary antibodies were $\alpha$-mouse, $\alpha$-rabbit, or $\alpha$-rat horseradish peroxidase-conjugated, from GE Healthcare, and used at 1:1000 dilution in 4\% milk in PBST. Blots were then washed again with PBST and ECL substrate from Thermo Scientific used to visualize the proteins.

\section{Optical trapping}

The optical trapping was performed as described previously ${ }^{30,41}$, with some modification to the reaction buffer and with the addition of flow. Bead conjugation and microtubule seed preparation were as previously described. Polystyrene beads conjugated to anti-His antibodies were incubated with Mps1-1 kinetochores at a concentration of $9.0 \mathrm{nM}$ Dsn1 for 1 hour at $4{ }^{\circ} \mathrm{C}$. This concentration is $50 \%$ higher than in our previous force-clamping experiments and potentially resulted in multiple kinetochore particles binding to a single microtubule. Reaction chambers were constructed on glass slides using sodium hydroxide- and ethanol-cleaned coverslips for an approximately $14 \mu \mathrm{L}$ volume chamber. Coverslip-anchored microtubules were grown via successive introduction of: biotinylated bovine serum albumin, BRB80, avidin DN, BRB80, GMPCPP-stabilized microtubule seeds, GTP- and $\kappa$-casein-containing BRB80, and then a final reaction buffer consisting of BRB80, $0.3 \mathrm{mg} / \mathrm{mL} \kappa$-casein, $1 \mathrm{mM} \mathrm{GTP,} \mathrm{2 \%} \mathrm{pluronic,} 0.8 \mathrm{mM}$ DTT, $48 \mathrm{mM}$ glucose, $1.6 \mathrm{mg} / \mathrm{mL}$ glucose oxidate, $0.3 \mathrm{mg} / \mathrm{mL}$ catalase, $3 \mathrm{nM}$ purified Dam1 complex, $400 \mu \mathrm{M}$ ATP, bead-bound kinetochores, and $2.6 \mathrm{mg} / \mathrm{mL}$ unpolymerized tubulin. This is similar to previous versions of the optical trap assay except with lower casein and with the addition of pluronic and ATP. Additionally, the trap stiffness was set to the lowest possible level, 0.045 $\mathrm{pN} / \mathrm{nm}$, in order to minimize extra material becoming caught in the trap. During an experiment, 

323 described $^{53}$. author upon request.

\section{Data availability}

\section{Acknowledgements}

the laser was used to manipulate one bead to bind to the side of a dynamic microtubule tip. Then the bead was clamped at $5 \mathrm{pN}$ of force and dragged to the tip. After 90 seconds of tracking with the tip at $5 \mathrm{pN}$, the force was either dropped to $1 \mathrm{pN}$, left at $5 \mathrm{pN}$, or elevated to $8 \mathrm{pN}$. Continued tip tracking was confirmed at the new force ( 20 seconds) and then $20 \mu \mathrm{L}$ of kinase-containing flow buffer was introduced via capillary action. Flow buffer contained BRB80, $0.3 \mathrm{mg} / \mathrm{mL} \kappa-$ casein, $1 \mathrm{mM}$ GTP, $2 \%$ pluronic, $0.8 \mathrm{mM}$ DTT, $48 \mathrm{mM}$ glucose, $1.6 \mathrm{mg} / \mathrm{mL}$ gluocose oxidate, 0.3 $\mathrm{mg} / \mathrm{mL}$ catalase, $500 \mu \mathrm{M}$ ATP, $2.4 \mathrm{mg} / \mathrm{mL}$ tubulin and $8.6 \mu \mathrm{M}$ AurB* or AurB*-KD. Flow in the chamber occurred immediately and was observed in the DIC view on the microscope. Events were observed without interference until either a rupture of the attachment occurred, or the event was ended by a second bead falling into the trap or by the bead tracking permanently back to the microtubule seed. Detachment rates were calculated by dividing the total number of detachments observed under a given condition by the total attachment time recorded. Uncertainties were estimated by dividing the detachment rate by the square root of the number of detachments. Kaplan-Meier analyses, including error analysis, were performed by Prism (GraphPad). The rupture force assay for Fig. S3a was performed under conditions identical to those previously

The authors declare that the main data supporting the findings of this study are available within the article and its Supplementary Information files. Extra data are available from the corresponding 
331 We thank Arshad Desai for antibodies. We also thank Trisha Davis, Hironori Funabiki and

332 members of the S.B. and C.L.A. labs for critical reading of the manuscript. A.K.D. is a Postdoctoral

333 Fellow of the American Cancer Society. This work was supported by that fellowship, Packard

334 Fellowship 2006-30521 (to C.L.A.), and NIH grants R01GM079373 (to C.L.A) and

335 R01GM064386 (to S.B.). S.B. is also an investigator of the Howard Hughes Medical Institute.

337 Author Contributions

338 A.K.D. conceptually designed and performed experiments, analyzed the data, and wrote the 339 manuscript; C.L.A. and S.B. conceptually designed experiments, analyzed the data, and wrote the 340 manuscript with A.K.D.

341

342 Competing Interests

343 The authors declare no competing interests.

345 References

3461 Cheeseman, I. M. \& Desai, A. Molecular architecture of the kinetochore-microtubule 347 interface. Nature reviews. Molecular cell biology 9, 33-46, doi:10.1038/nrm2310 (2008).

3482 Santaguida, S. \& Musacchio, A. The life and miracles of kinetochores. The EMBO journal $349 \quad$ 28, 2511-2531, doi:10.1038/emboj.2009.173 (2009).

3503 Nicklas, R. B. \& Koch, C. A. Chromosome micromanipulation. 3. Spindle fiber tension 351 and the reorientation of mal-oriented chromosomes. The Journal of cell biology 43, 40-50 (1969). 
3534 Nicklas, R. B. \& Ward, S. C. Elements of error correction in mitosis: microtubule capture, release, and tension. The Journal of cell biology 126, 1241-1253 (1994).

3555 Biggins, S. et al. The conserved protein kinase Ipl1 regulates microtubule binding to $356 \quad$ kinetochores in budding yeast. Genes Dev 13, 532-544 (1999).

3576 Tanaka, T. U. et al. Evidence that the Ipl1-Sli15 (Aurora kinase-INCENP) complex 358 promotes chromosome bi-orientation by altering kinetochore-spindle pole connections. $359 \quad$ Cell 108, 317-329 (2002).

3607 Lampson, M. A., Renduchitala, K., Khodjakov, A. \& Kapoor, T. M. Correcting improper 361 chromosome-spindle attachments during cell division. Nat Cell Biol 6, 232-237, 362 doi:10.1038/ncb1102 (2004).

3638 Cheeseman, I. M., Chappie, J. S., Wilson-Kubalek, E. M. \& Desai, A. The conserved KMN 364 network constitutes the core microtubule-binding site of the kinetochore. Cell 127, 983365 997, doi:10.1016/j.cell.2006.09.039 (2006).

3669 Cimini, D., Wan, X., Hirel, C. B. \& Salmon, E. D. Aurora kinase promotes turnover of 367 kinetochore microtubules to reduce chromosome segregation errors. Current biology : CB $368 \quad$ 16, 1711-1718, doi:10.1016/j.cub.2006.07.022 (2006).

36910 DeLuca, J. G. et al. Kinetochore microtubule dynamics and attachment stability are 370 regulated by Hec1. Cell 127, 969-982, doi:10.1016/j.cell.2006.09.047 (2006).

$371 \quad 11$ Kelly, A. E. \& Funabiki, H. Correcting aberrant kinetochore microtubule attachments: an 372 Aurora B-centric view. Current opinion in cell biology 21, 51-58, 373 doi:10.1016/j.ceb.2009.01.004 (2009). 
37412 Liu, D., Vader, G., Vromans, M. J., Lampson, M. A. \& Lens, S. M. Sensing chromosome

375

376

377

378

379

380

381

382 bi-orientation by spatial separation of aurora B kinase from kinetochore substrates. Science (New York, N.Y.) 323, 1350-1353, doi:10.1126/science.1167000 (2009).

13 Welburn, J. P. et al. Aurora B phosphorylates spatially distinct targets to differentially regulate the kinetochore-microtubule interface. Molecular cell 38, 383-392, doi:10.1016/j.molcel.2010.02.034 (2010).

14 Yue, Z. et al. Deconstructing Survivin: comprehensive genetic analysis of Survivin function by conditional knockout in a vertebrate cell line. The Journal of cell biology 183, 279-296, doi:10.1083/jcb.200806118 (2008).

15 Campbell, C. S. \& Desai, A. Tension sensing by Aurora B kinase is independent of survivin-based centromere localization. Nature 497, 118-121, doi:10.1038/nature12057 (2013).

16 Hengeveld, R. C. C., Vromans, M. J. M., Vleugel, M., Hadders, M. A. \& Lens, S. M. A. Inner centromere localization of the CPC maintains centromere cohesion and allows mitotic checkpoint silencing. Nature communications $\mathbf{8 ,} \quad 15542$, doi:10.1038/ncomms15542 (2017).

17 Katayama, H., Brinkley, W. R. \& Sen, S. The Aurora kinases: role in cell transformation and tumorigenesis. Cancer metastasis reviews 22, 451-464 (2003).

18 Katayama, H. et al. Mitotic kinase expression and colorectal cancer progression. Journal of the National Cancer Institute 91, 1160-1162 (1999).

19 Tatsuka, M. et al. Multinuclearity and increased ploidy caused by overexpression of the aurora- and Ipl1-like midbody-associated protein mitotic kinase in human cancer cells. Cancer research 58, 4811-4816 (1998). 
39720 Lens, S. M. et al. Uncoupling the central spindle-associated function of the chromosomal $398 \quad$ passenger complex from its role at centromeres. Molecular biology of the cell 17, 18971909, doi:10.1091/mbc.e05-08-0727 (2006).

40021 Ruchaud, S., Carmena, M. \& Earnshaw, W. C. Chromosomal passengers: conducting cell division. Nature reviews. Molecular cell biology 8, 798-812, doi:10.1038/nrm2257 (2007).

40222 Adams, R. R. et al. INCENP binds the Aurora-related kinase AIRK2 and is required to 403 target it to chromosomes, the central spindle and cleavage furrow. Current biology : $C B$ 10, $1075-1078$ (2000).

405

23 Bishop, J. D. \& Schumacher, J. M. Phosphorylation of the carboxyl terminus of inner centromere protein (INCENP) by the Aurora B Kinase stimulates Aurora B kinase activity. The Journal of biological chemistry 277, 27577-27580, doi:10.1074/jbc.C200307200 (2002).

40924 Bolton, M. A. et al. Aurora B kinase exists in a complex with survivin and INCENP and its kinase activity is stimulated by survivin binding and phosphorylation. Molecular biology of the cell 13, 3064-3077, doi:10.1091/mbc.E02-02-0092 (2002).

41225 Honda, R., Korner, R. \& Nigg, E. A. Exploring the functional interactions between Aurora B, INCENP, and survivin in mitosis. Molecular biology of the cell 14, 3325-3341, doi:10.1091/mbc.E02-11-0769 (2003).

41526 Kaitna, S., Mendoza, M., Jantsch-Plunger, V. \& Glotzer, M. Incenp and an aurora-like kinase form a complex essential for chromosome segregation and efficient completion of cytokinesis. Current biology : CB 10, 1172-1181 (2000). 
41827 Kang, J. et al. Functional cooperation of Dam1, Ipl1, and the inner centromere protein

419 (INCENP)-related protein Sli15 during chromosome segregation. The Journal of cell biology 155, 763-774, doi:10.1083/jcb.200105029 (2001).

42128 Wang, E., Ballister, E. R. \& Lampson, M. A. Aurora B dynamics at centromeres create a diffusion-based phosphorylation gradient. The Journal of cell biology 194, 539-549, doi:10.1083/jcb.201103044 (2011).

42429 Umbreit, N. T. et al. The Ndc80 kinetochore complex directly modulates microtubule dynamics. Proceedings of the National Academy of Sciences of the United States of 42730 Sarangapani, K. K., Akiyoshi, B., Duggan, N. M., Biggins, S. \& Asbury, C. L. America 109, 16113-16118, doi:10.1073/pnas.1209615109 (2012). Phosphoregulation promotes release of kinetochores from dynamic microtubules via 43131 Smith, C. A., McAinsh, A. D. \& Burroughs, N. J. Human kinetochores are swivel joints Cell 137, 672-684, doi:10.1016/j.cell.2009.03.035 (2009).

43533 Tien, J. F. et al. Kinetochore biorientation in Saccharomyces cerevisiae requires a tightly folded conformation of the Ndc80 complex. Genetics 198, 1483-1493, doi:10.1534/genetics.114.167775 (2014).

43834 Joglekar, A. P., Bloom, K. \& Salmon, E. D. In vivo protein architecture of the eukaryotic kinetochore with nanometer scale accuracy. Curr Biol 19, 694-699, doi:10.1016/j.cub.2009.02.056 (2009). 
44135 Campbell, I. D. \& Humphries, M. J. Integrin structure, activation, and interactions. Cold

$442 \quad$ Spring Harbor perspectives in biology 3, doi:10.1101/cshperspect.a004994 (2011).

$443 \quad 36$ Ranade, S. S., Syeda, R. \& Patapoutian, A. Mechanically Activated Ion Channels. Neuron $444 \quad$ 87, 1162-1179, doi:10.1016/j.neuron.2015.08.032 (2015).

44537 Liu, D. et al. Regulated targeting of protein phosphatase 1 to the outer kinetochore by 446 KNL1 opposes Aurora B kinase. The Journal of cell biology 188, 809-820, 447 doi:10.1083/jcb.201001006 (2010).

44838 Foley, E. A., Maldonado, M. \& Kapoor, T. M. Formation of stable attachments between 449 kinetochores and microtubules depends on the B56-PP2A phosphatase. Nature cell biology 13, 1265-1271, doi:10.1038/ncb2327 (2011).

45139 Suijkerbuijk, S. J., Vleugel, M., Teixeira, A. \& Kops, G. J. Integration of kinase and 452 phosphatase activities by BUBR1 ensures formation of stable kinetochore-microtubule attachments. Developmental cell 23, 745-755, doi:10.1016/j.devcel.2012.09.005 (2012).

45440 Kruse, T. et al. Direct binding between BubR1 and B56-PP2A phosphatase complexes 455 regulate mitotic progression. Journal of cell science 126, 1086-1092, 456 doi:10.1242/jcs.122481 (2013).

45741 Akiyoshi, B. et al. Tension directly stabilizes reconstituted kinetochore-microtubule 458 attachments. Nature 468, 576-579, doi:10.1038/nature09594 (2010).

45942 London, N., Ceto, S., Ranish, J. A. \& Biggins, S. Phosphoregulation of Spc105 by Mps1 and PP1 regulates Bub1 localization to kinetochores. Current biology : CB 22, 900-906, doi:10.1016/j.cub.2012.03.052 (2012). 
46243 Meyer, R. E. et al. Mps1 and Ipl1/Aurora B act sequentially to correctly orient chromosomes on the meiotic spindle of budding yeast. Science (New York, N.Y.) 339, 10711074, doi:10.1126/science.1232518 (2013).

465

466

467

468

469

470

$471 \quad 46$

472

473

$474 \quad 47$

475

476

$477 \quad 48$

478

479

480

481

$482 \quad 50$

483

Akiyoshi, B., Nelson, C. R., Ranish, J. A. \& Biggins, S. Analysis of Ipl1-mediated phosphorylation of the $\mathrm{Ndc} 80$ kinetochore protein in Saccharomyces cerevisiae. Genetics 183, 1591-1595, doi:10.1534/genetics.109.109041 (2009).

45 Akiyoshi, B., Nelson, C. R. \& Biggins, S. The aurora B kinase promotes inner and outer kinetochore interactions in budding yeast. Genetics 194, 785-789, doi:10.1534/genetics.113.150839 (2013).

46 Hauf, S. et al. The small molecule Hesperadin reveals a role for Aurora B in correcting kinetochore-microtubule attachment and in maintaining the spindle assembly checkpoint. The Journal of cell biology 161, 281-294, doi:10.1083/jcb.200208092 (2003).

47 Cheeseman, I. M. et al. Implication of a novel multiprotein Dam1p complex in outer kinetochore function. The Journal of cell biology 155, 1137-1145, doi:10.1083/jcb.200109063 (2001).

48 Ciferri, C. et al. Implications for kinetochore-microtubule attachment from the structure of an engineered Ndc80 complex. Cell 133, 427-439, doi:10.1016/j.cell.2008.03.020 (2008).

49 Gestaut, D. R. et al. Phosphoregulation and depolymerization-driven movement of the Dam1 complex do not require ring formation. Nature cell biology 10, 407-414, doi:10.1038/ncb1702 (2008).

Kalantzaki, M. et al. Kinetochore-microtubule error correction is driven by differentially regulated interaction modes. Nat Cell Biol 17, 421-433, doi:10.1038/ncb3128 (2015). 
$48451 \quad$ Umbreit, N. T. et al. Kinetochores require oligomerization of Dam1 complex to maintain microtubule attachments against tension and promote biorientation. Nature communications 5, 4951, doi:10.1038/ncomms5951 (2014).

48752 Sarangapani, K. K. et al. Sister kinetochores are mechanically fused during meiosis I in yeast. Science (New York, N.Y.) 346, 248-251, doi:10.1126/science.1256729 (2014).

53 Miller, M. P., Asbury, C. L. \& Biggins, S. A TOG Protein Confers Tension Sensitivity to doi:10.1016/j.cell.2016.04.030 (2016).

54 Sandall, S. et al. A Bir1-Sli15 complex connects centromeres to microtubules and is required to sense kinetochore tension. Cell 127, 1179-1191, doi:10.1016/j.cell.2006.09.049 (2006). science 124, 622-634, doi:10.1242/jcs.072629 (2011). kinetochore proteins by promoting Aurora B kinase activity. The Journal of cell biology

50157 Cormier, A., Drubin, D. G. \& Barnes, G. Phosphorylation regulates kinase and microtubule binding activities of the budding yeast chromosomal passenger complex in vitro. The Journal of biological chemistry 288, 23203-23211, doi:10.1074/jbc.M113.491480 (2013). 
proteins. Protein expression and purification 83, 217-225, doi:10.1016/j.pep.2012.04.006

507

508

509

510

511

512

513

514

515

516

517

518

519

520

521

522

523

524

525

526

527

528

529

530

531

532

533

534

535

536

537

538

539

540

541

542
(2012).

59 Tropea, J. E., Cherry, S. \& Waugh, D. S. Expression and purification of soluble His(6)tagged TEV protease. Methods in molecular biology (Clifton, N.J.) 498, 297-307, doi:10.1007/978-1-59745-196-3_19 (2009).

\section{Figure Legends}

Fig. 1 | An Aurora B kinase phosphorylates kinetochores. a AurB* was created by fusing the C-terminal activation box of INCENP ${ }^{\text {Sli15 }}$ to Aurora $\mathrm{B}^{\mathrm{Ipl1}}$. b Schematic for the experiments shown in (c)-(e). c Mps1-1 kinetochores (purified from SBY8726) were phosphorylated by either $0.2 \mu \mathrm{M}$ AurB* or $0.2 \mu \mathrm{M}$ AurB*-KD and ${ }^{32} \mathrm{P}-\gamma$-labeled ATP and then visualized by silver stain and autoradiography. The first two lanes show AurB* or AurB*-KD alone with no kinetochores after 5 minutes of incubation, the next five lanes show a time course of kinetochores incubated with AurB*, and each of the last two lanes show kinetochores incubated with either no kinase or AurB*KD for 3.5 minutes. The bands of relevant Aurora B substrates are labeled. Positions of protein standards are shown on the left in kDa. d Quantification of $N d c 80$ incorporation of ${ }^{32} \mathrm{P}$ in $(\mathbf{c})$. Red points (AurB*) are from the time course, the blue point (ATP only) is from the second to last lane, and the grey point (AurB*-KD) is from the last lane. Units are arbitrary and relative. e Wild type (SBY8253) or Ndc80-7A (SBY8522) kinetochores (KT) were incubated with either buffer or 1 $\mu \mathrm{M}$ AurB* and ${ }^{32} \mathrm{P}-\gamma$-labeled ATP for 3 minutes. A decrease in phosphorylation in the Ndc 80 band when comparing lanes 2 and 4 show that AurB* is phosphorylating Ndc 80 on these seven known sites.

\section{Fig. 2 | Aurora B phosphorylation is sufficient for kinetochores' release of microtubules. a} Schematic for the experiment shown in (b). b Purified wild-type (WT) (SBY8253) or Ndc80-7A (SBY8522) kinetochores were bound to purified Dam1 complex (Dam1c; SBY12464) and then to taxol-stabilized fluorescently-labeled microtubules in the presence of ATP. Buffer or $1 \mu \mathrm{M}$ AurB* was then added and components retained after a wash were separated via SDS-PAGE and visualized by Western blot and fluorescence scan. $P$-Ndc80: phosphorylated Ndc80. c Quantification of bound microtubules (lowest row in (b)) presented as the average of three independent experiments; error bars represent standard deviations. Values were normalized to microtubules bound at $\mathrm{t}=0$. $\mathrm{P}$ values between the $\mathrm{t}=20$ timepoints using an unpaired $\mathrm{t}$-test are as follows: $\quad 0.0054 \quad(\mathrm{WT}+$ buffer/WT+AurB $*), \quad 0.16 \quad(\mathrm{WT}+\mathrm{buffer} / 7 \mathrm{~A}+\mathrm{AurB} *), \quad 0.033$ $(\mathrm{WT}+\mathrm{AurB} * / 7 \mathrm{~A}+\mathrm{AurB} *)$.

Fig. 3 | A flow assay introduces Aurora B to a kinetochore-microtubule attachment under tension. a Schematic of the optical trap flow assay. Tension across kinetochore-microtubule 
attachments is proportional to the displacement of the bead from the center of the laser trap. b, c Traces of optical trap data for two individual events. Gray points show the force applied and green points show the same data after smoothing. Blue traces show the relative position of the stage over time. Kinase introduction occurred at time 0 seconds; blue shading indicates data before kinase introduction. (b) shows an event that ended when a second bead became caught in the laser trap and corresponds to Movie 1. (c) shows an event that ended in a detachment and corresponds to Movie 2. The yellow ball indicates when detachment occurred.

Fig. 4 | Aurora B phosphorylation is sufficient to release kinetochores from the tips of dynamic microtubules. a Each individual event, corresponding to one bead, graphed as a bar whose length corresponds to its total time. Events that ended in detachment are marked with yellow balls. Events that were interrupted are unmarked. Asterisks indicate the events shown in Figs. 3bc. b Kaplan-Meier survival analysis for flow with AurB*-KD or AurB*. AurB*-KD was flowed in at a final concentration of $5 \mu \mathrm{M}$ and AurB* was flowed in at a final concentration of either 0.5 or $5 \mu \mathrm{M}$. Detachment rates at these concentrations of AurB* were indistinguishable from one another and were therefore pooled. Ticks indicate censored data points from interrupted events that did not end in detachment. Shaded errors show standard error. $\mathrm{P}=0.014$ using a log-rank test. c Detachment rates of kinetochores from microtubules held under $1.3 \pm 0.6 \mathrm{pN}$ or $1.1 \pm 0.5 \mathrm{pN}$ of force after flow with AurB*-KD or AurB*, respectively. Error bars represent uncertainty due to counting statistics $(n=26$ beads and 7 detachments (AurB*-KD) and $n=28$ beads and 18 detachments (AurB*)).

Fig. 5 | Tension does not suppress Aurora B-triggered detachment. a Detachment rates of kinetochores from microtubules at different forces with introduction of either $5 \mu \mathrm{M}$ AurB* or 5 $\mu \mathrm{M}$ AurB*-KD. Some data from Fig. 4 are replotted here. b Kaplan-Meier survival analysis for flow with $5 \mu \mathrm{M}$ AurB*-KD, $0.5 \mu \mathrm{M}$ AurB*, or $5 \mu \mathrm{M}$ AurB*. Ticks represent censored data points from interrupted events that did not end in detachment. Shaded errors show standard error. P values using a log-rank test are as follows: 0.040 (KD vs $0.5 \mu \mathrm{M}$ AurB $\left.{ }^{*}\right), 0.0097(0.5 \mu \mathrm{M}$ vs $5 \mu \mathrm{M}$ AurB*), $<0.0001$ (KD vs $5 \mu \mathrm{M}$ AurB*). c Detachment rates of kinetochores from microtubules at different levels of AurB* kinase activity, where AurB*-KD is $0 \mu \mathrm{M}$ AurB*. Kinetochores were held at $4.7 \pm 0.6 \mathrm{pN}\left(\mathrm{AurB}^{*}-\mathrm{KD}\right), 4.8 \pm 0.6 \mathrm{pN}\left(0.5 \mu \mathrm{M} \mathrm{AurB}^{*}\right)$, or $5.0 \pm 0.2 \mathrm{pN}(5 \mu \mathrm{M}$ AurB*). Error bars represent uncertainty due to counting statistics $(n=20$ events and 11 detachments (AurB*-KD), $\mathrm{n}=4$ events and 4 detachments $\left(0.5 \mu \mathrm{M}\right.$ AurB $\left.{ }^{*}\right)$, or $\mathrm{n}=12$ events and 8 detachments $(5 \mu \mathrm{M}$ AurB*)).

Fig. S1 | Immunoblot of purified kinetochores from either wild-type (SBY8253) or Mps1-1 (SBY8726) kinetochores showing that Mps1-1 kinetochores lack co-purifying Dam1 complex. 
585 Fig. S3 | a Rupture force values for various kinetochores detaching from microtubules as 586 determined using an optical force-ramp. Where indicated, exogenously purified Dam 1 complex 587 (Dam1c) and/or ATP were included in the trapping buffer. b Traces of optical trap data for an 588 individual force-clamp event. Gray points show the force applied across the kinetochore589 microtubule attachment and green points show the same data after smoothing. The blue trace 590 shows the relative position of a single kinetochore-coupled bead as it tracks with the microtubule 591 tip.

592

Fig. S4 | a Detachment rates for Mps1-1 kinetochores with Dam1 complex present, separated by concentration of kinase. The data at $1 \mathrm{pN}$ and $5 \mathrm{pN}$ are replotted from Figs. 4 and 5. For the data at $8 \mathrm{pN}, \mathrm{n}=4$ events and 2 detachments (AurB*-KD) or $\mathrm{n}=3$ events and 3 detachments $(5 \mu \mathrm{M}$ AurB*). b Detachment rates for wild-type kinetochores at high force. The assay was performed as diagramed in Fig. 3a, except with wild-type kinetochores and no addition of Dam1 complex. $5 \mu \mathrm{M}$ of either AurB* or AurB*-KD was flowed in.

Fig. S5 $\mid \mathbf{a}$ and b Kinetochore detachment rates measured specifically during microtubule growth (a) and during microtubule shortening (b). Rates measured for this work, using Mps1-1 602 kinetochores with Dam1 complex and AurB*-KD flow, are shown in gray. Rates for wild-type 603 kinetochores, from previous work ${ }^{41,53}$, are shown in green.

Movie 1 | Differential interference contrast (DIC) view of an event during the flow assay that ends in an interruption, with a second bead falling into the trap; this event corresponds to the traces in

607 Fig. 3b. Timer counts down until flow is introduced and then counts up. The second bead appears $608 \quad 2: 23$ after flow initiation.

Movie 2 | Differential interference contrast (DIC) view of an event during the flow assay that ends in a detachment; this event corresponds to the traces in Fig. 3c. Timer counts down until flow is introduced and then counts up. Detachment occurs at 5:12. 
bioRxiv preprint doi: https://doi.org/10.1101/415992; this version posted September 13, 2018. The copyright holder for this preprint (which was Figure $\mathbf{1}$

not certified by peer review) is the author/funder, who has granted bioRxiv a license to display the preprint in perpetuity. It is made available

a

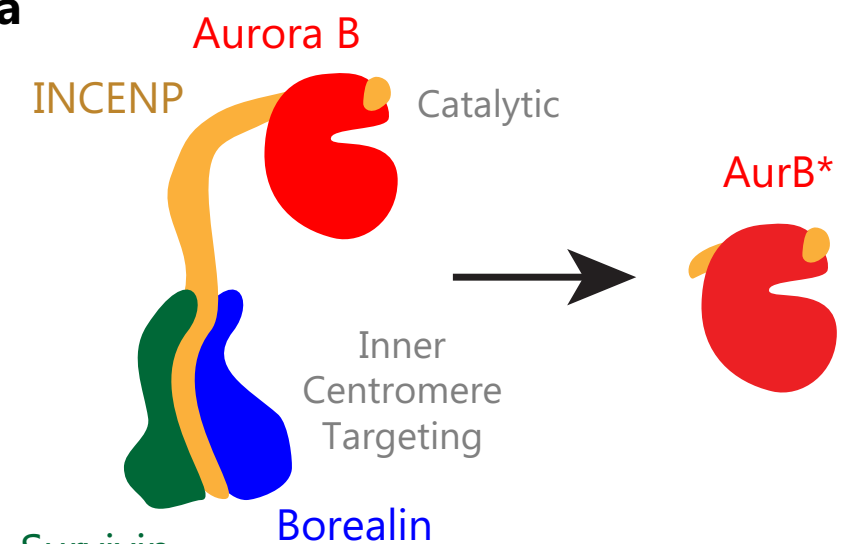

b

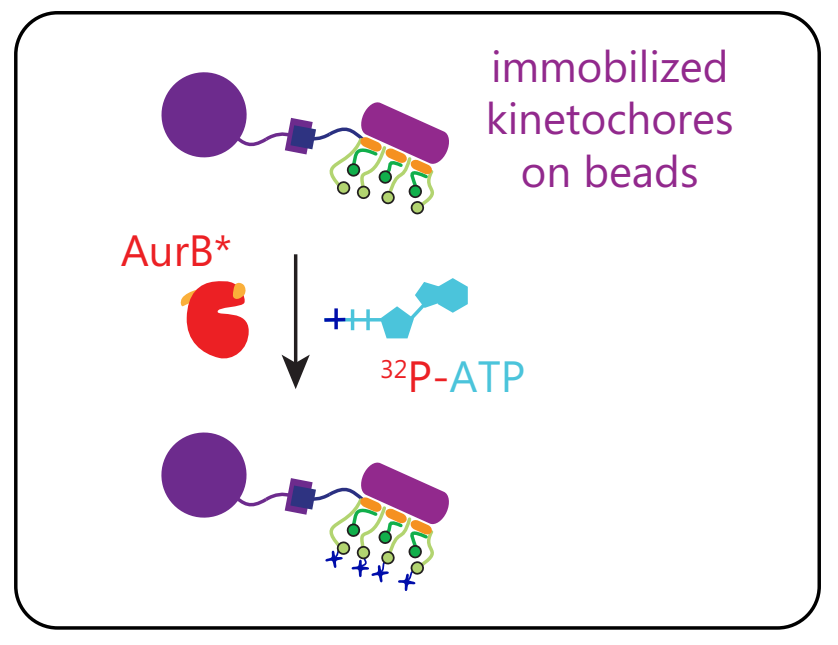

C
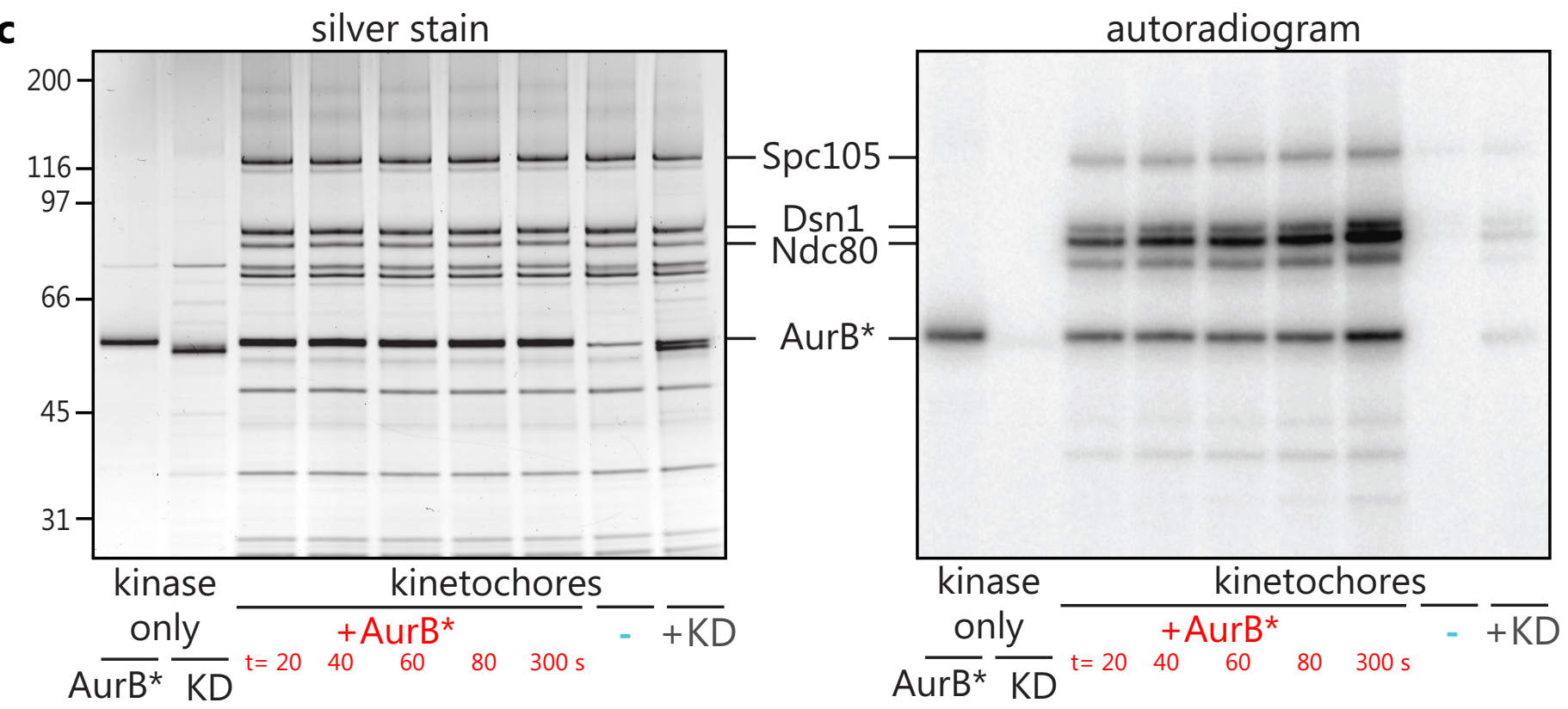

d

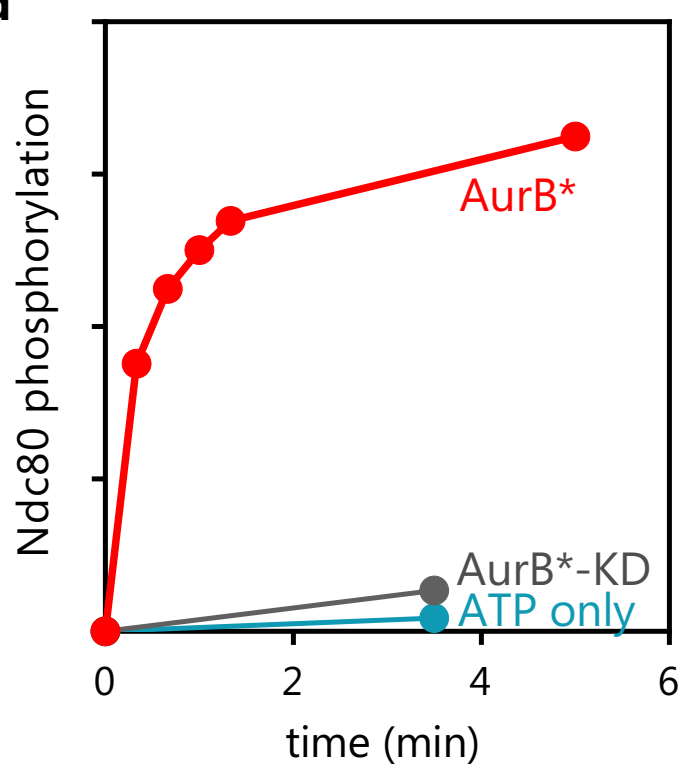

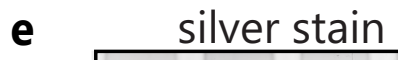

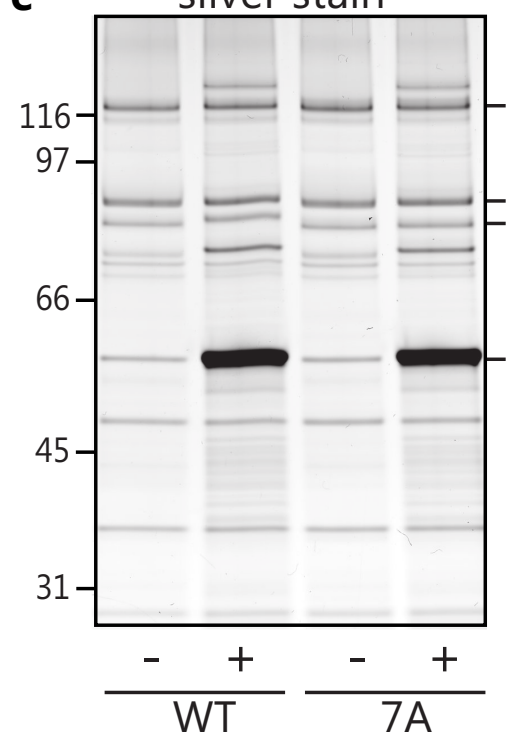

autoradiogram

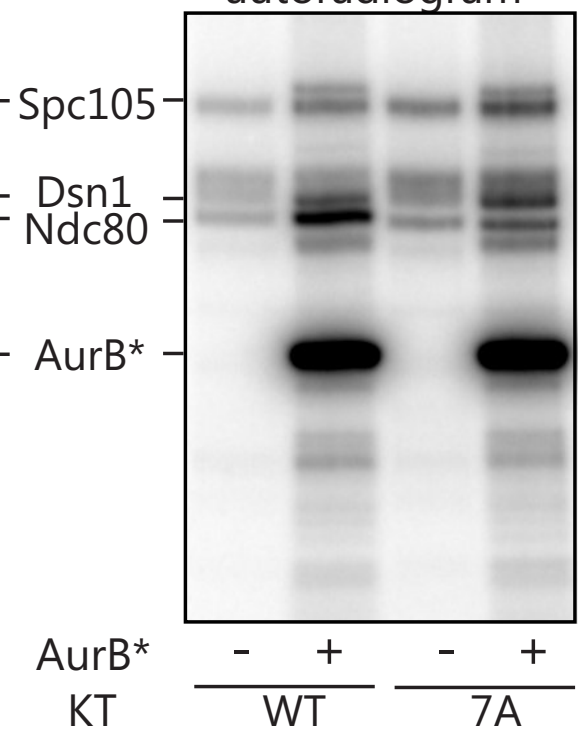


Fig. 1 | An Aurora B kinase phosphorylates kinetochores. a AurB* was created by fusing the C-terminal activation box of INCENP ${ }^{\text {Sli15 }}$ to Aurora $B^{\text {Ipl1 }}$. b Schematic for the experiments shown in (c)-(e). c Mps1-1 kinetochores (purified from SBY8726) were phosphorylated by either $0.2 \mu \mathrm{M}$ AurB* or $0.2 \mu \mathrm{M}$ AurB*-KD and ${ }^{32} \mathrm{P}-\nu$-labeled ATP and then visualized by silver stain and autoradiography. The first two lanes show AurB* or AurB*-KD alone with no kinetochores after 5 minutes of incubation, the next five lanes show a timecourse of kinetochores incubated with Aur $B^{*}$, and each of the last two lanes show kinetochores incubated with either no kinase or AurB*-KD for 3.5 minutes. The bands of relevant Aurora $B$ substrates are labeled. Positions of protein standards are shown on the left in $\mathrm{kDa}$. $\mathbf{d}$ Quantification of $\mathrm{Ndc} 80$ incorporation of ${ }^{32} \mathrm{P}$ in (c). Red points (AurB*) are from the timecourse, the blue point (ATP only) is from the second to last lane, and the grey point $\left(A u r B^{*}-K D\right)$ is from the last lane. Units are arbitrary and relative. e Wild type (SBY8253) or Ndc80-7A (SBY8522) kinetochores (KT) were incubated with either buffer or $1 \mu \mathrm{M}$ AurB* and ${ }^{32} \mathrm{P}-\nu^{-l a b e l e d}$ ATP for 3 minutes. A decrease in phosphorylation in the Ndc80 band when comparing lanes 2 and 4 show that AurB* is phosphorylating $\mathrm{Ndc} 80$ on these seven known sites. 
a

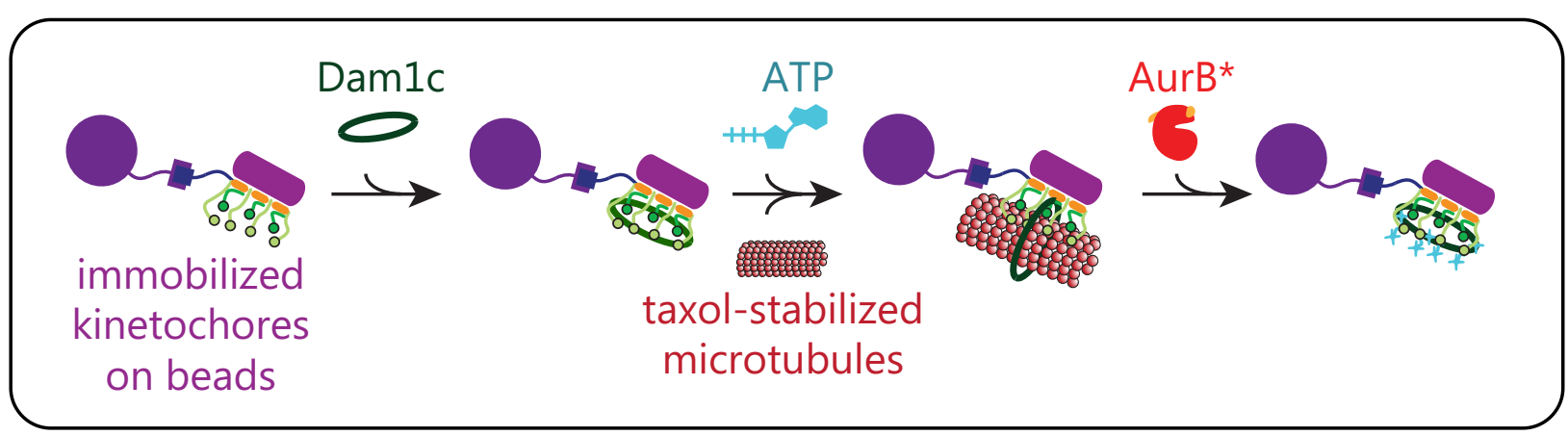

b

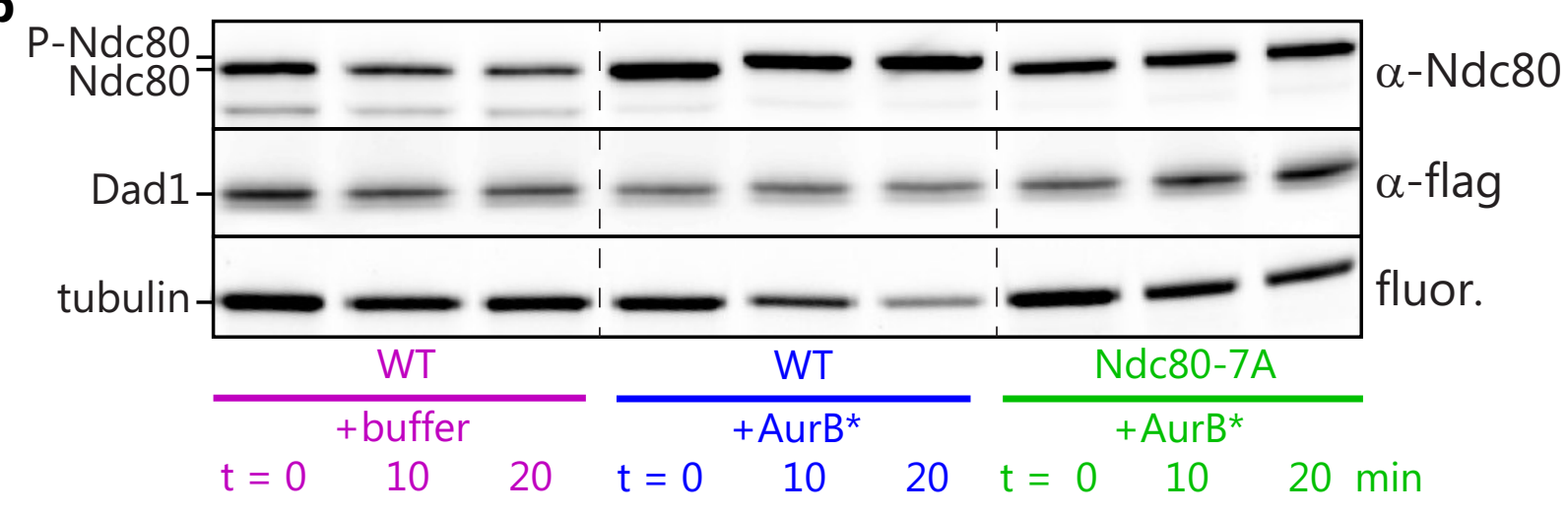

C

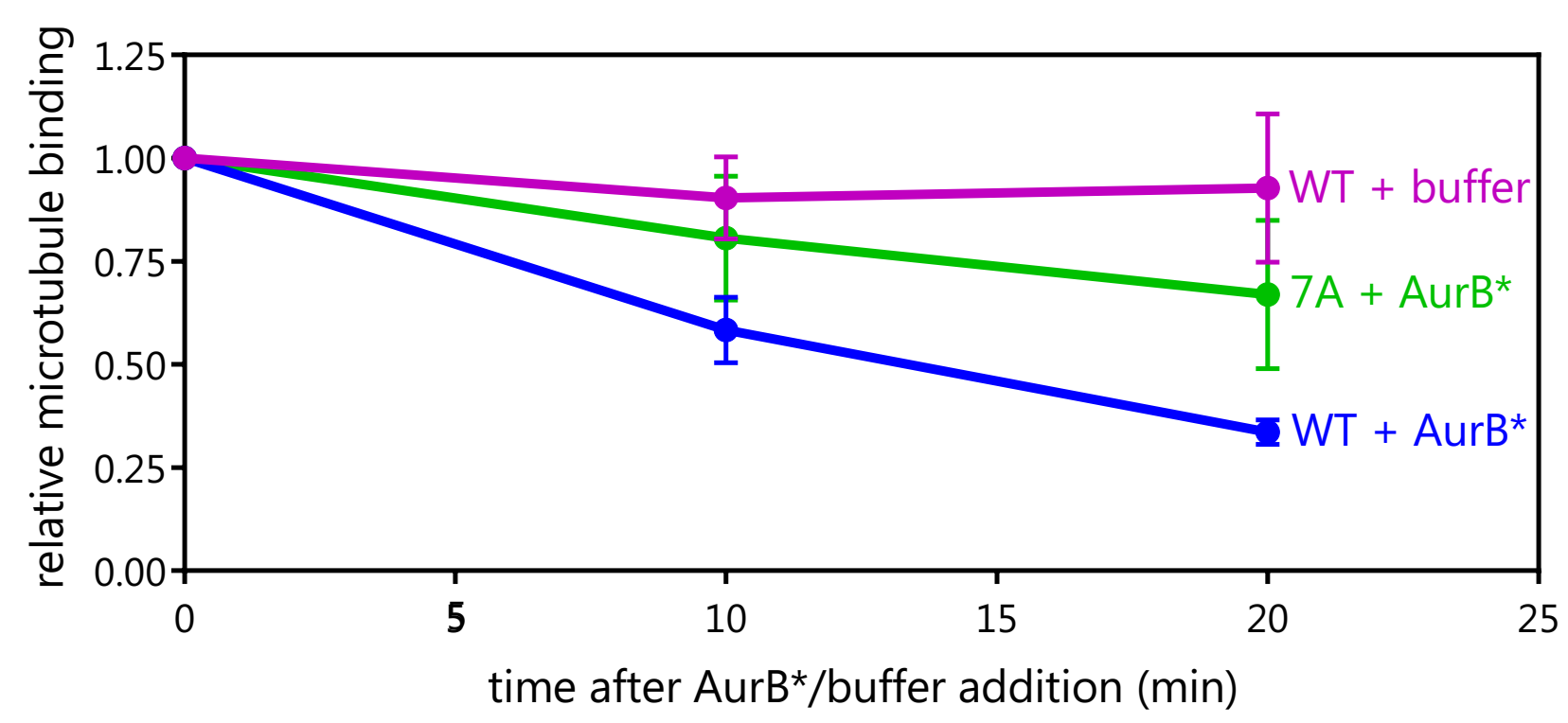

Fig. 2 | Aurora B phosphorylation is sufficient for kinetochores' release of microtubules. a Schematic for the experiment shown in (b). b Purified wild-type (WT) (SBY8253) or Ndc80-7A (SBY8522) kinetochores were bound to purified Dam1 complex (Dam1c; SBY12464) and then to taxol-stabilized fluorescently-labeled microtubules in the presence of ATP. Buffer or $1 \mu \mathrm{M}$ AurB* was then added and components retained after a wash were separated via SDSPAGE and visualized by Western blot and fluorescence scan. P-Ndc80: phosphorylated Ndc80. c Quantification of bound microtubules (lowest row in (b)) presented as the average of three independent experiments; error bars represent standard deviations. Values were normalized to microtubules bound at $t=0$. $P$ values between the $t=20$ timepoints using an unpaired t-test are as follows: $0.0054(\mathrm{WT}+$ buffer/WT+AurB*), $0.16(\mathrm{WT}+$ buffer/7A+AurB* $), 0.033$ $\left(\mathrm{WT}+\mathrm{AurB}^{*} / 7 \mathrm{~A}+\mathrm{AurB}{ }^{*}\right)$. 
a

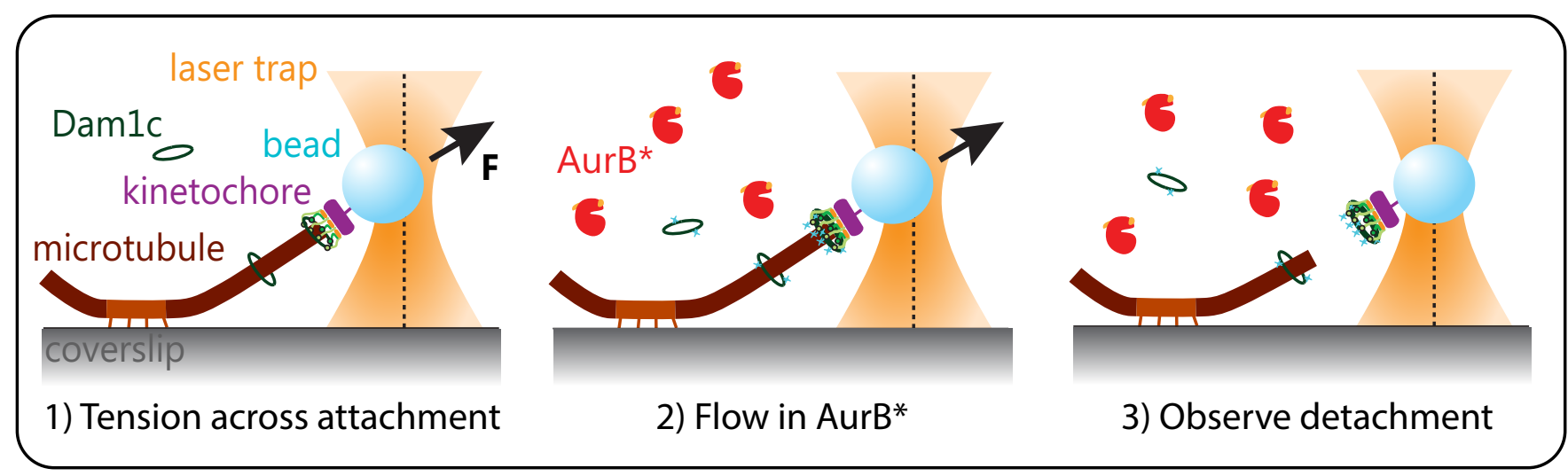

b
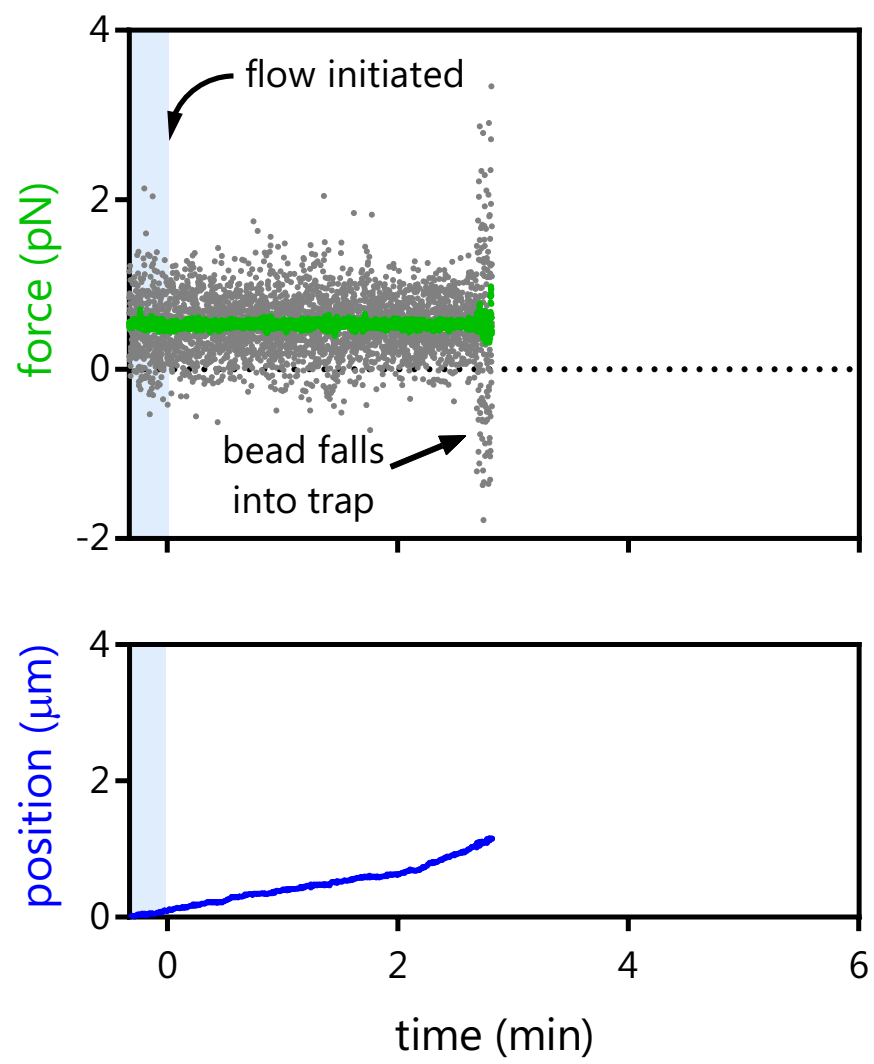

C
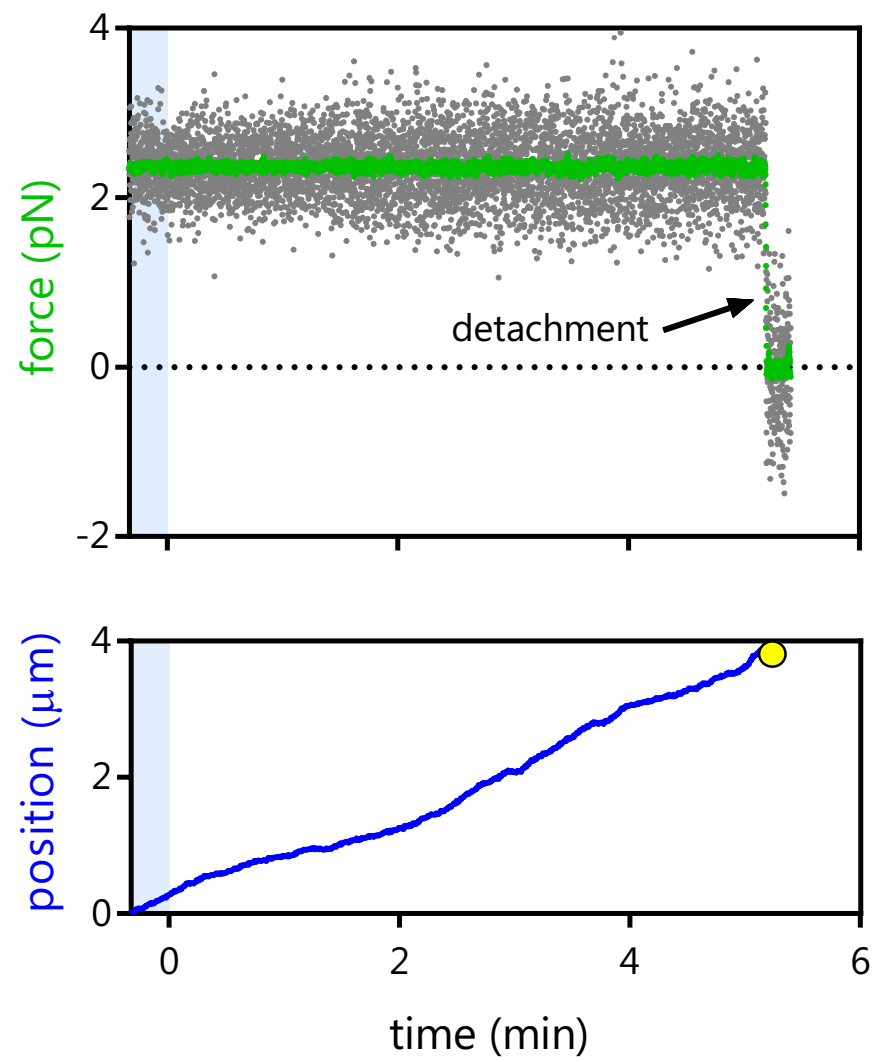

Fig. 3 | A flow assay introduces Aurora B to a kinetochore-microtubule attachment under tension. a Schematic of the optical trap flow assay. Tension across kinetochore-microtubule attachments is proportional to the displacement of the bead from the center of the laser trap. b, c Traces of optical trap data for two individual events. Gray points show the force applied and green points show the same data after smoothing. Blue traces show the relative position of the stage over time. Kinase introduction occurred at time 0 seconds; blue shading indicates data before kinase introduction. (b) shows an event that ended when a second bead became caught in the laser trap and corresponds to Movie 1. (c) shows an event that ended in a detachment and corresponds to Movie 2. The yellow ball indicates when detachment occurred. 

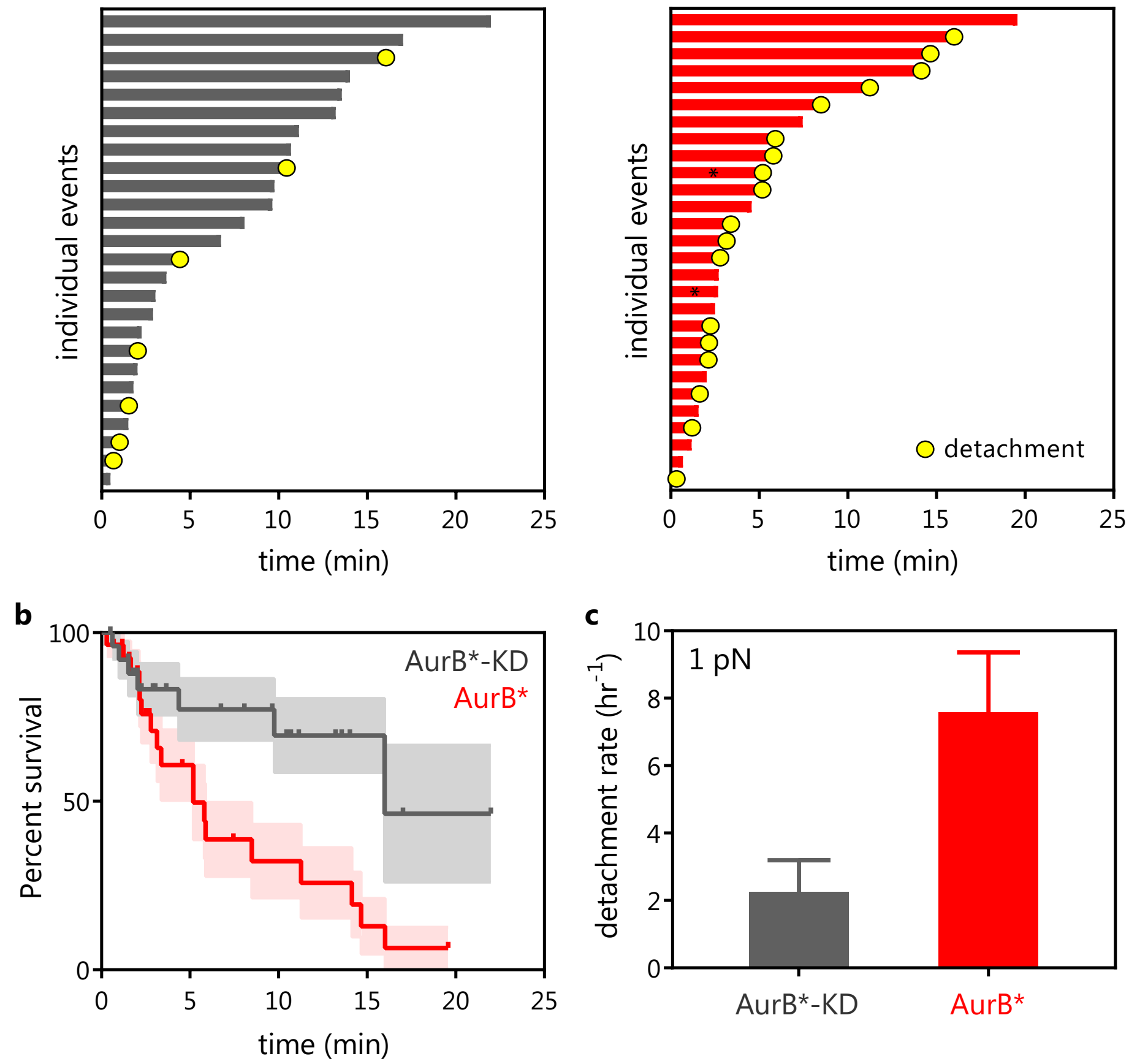

Fig. 4 | Aurora B phosphorylation is sufficient to release kinetochores from the tips of dynamic microtubules. a Each individual event, corresponding to one bead, graphed as a bar whose length corresponds to its total time. Events that ended in detachment are marked with yellow balls. Events that were interrupted are unmarked. Asterisks indicate the events shown in Figs. 3b-c. b Kaplan-Meier survival analysis for flow with AurB*-KD or AurB*. AurB* KD $^{*}$ was flowed in at a final concentration of $5 \mu \mathrm{M}$ and AurB* was flowed in at a final concentration of either 0.5 or $5 \mu \mathrm{M}$. Detachment rates at these concentrations of AurB* were indistinguishable from one another and were therefore pooled. Ticks indicate censored data points from interrupted events that did not end in detachment. Shaded errors show standard error. $\mathrm{P}=0.014$ using a log-rank test. $\mathrm{c}$ Detachment rates of kinetochores from microtubules held under $1.3 \pm 0.6 \mathrm{pN}$ or $1.1 \pm 0.5 \mathrm{pN}$ of force after flow with AurB ${ }^{*}-K D$ or AurB* ${ }^{*}$, respectively. Error bars represent uncertainty due to counting statistics ( $n=26$ beads and 7 detachments (AurB* $-K D)$ and $n=28$ beads and 18 detachments (AurB $\left.{ }^{*}\right)$ ). 

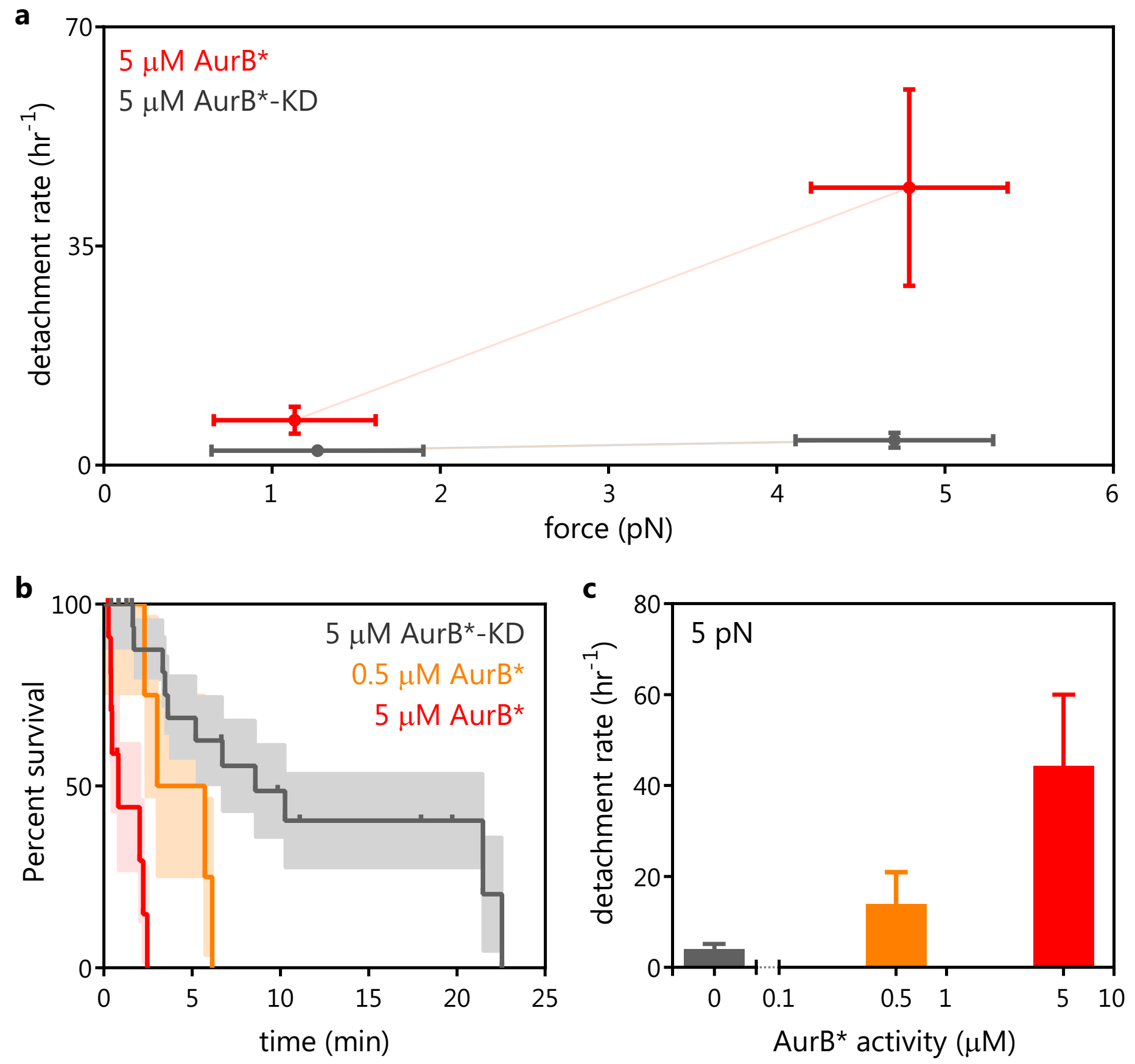

Fig. 5 | Tension does not suppress Aurora B-triggered detachment. a Detachment rates of kinetochores from microtubules at different forces with introduction of either $5 \mu \mathrm{M}$ AurB* or $5 \mu \mathrm{M}$ AurB*-KD. Some data from Fig. 4 are replotted here. b Kaplan-Meier survival analysis for flow with $5 \mu \mathrm{M}$ AurB* $-K D, 0.5 \mu \mathrm{M}$ AurB* ${ }^{*}$, or $5 \mu \mathrm{M}$ AurB* Ticks represent censored data points from interrupted events that did not end in detachment. Shaded errors show standard error. P values using a log-rank test are as follows: 0.040 (KD vs $0.5 \mu \mathrm{M}$ AurB*), $0.0097(0.5 \mu \mathrm{M}$ vs $5 \mu \mathrm{M}$ AurB*), $<0.0001$ ( $K D$ vs $5 \mu \mathrm{M}$ AurB*). c Detachment rates of kinetochores from microtubules at different levels of AurB* kinase activity, where AurB ${ }^{*}-K D$ is $0 \mu \mathrm{M}$ AurB* . Kinetochores were held at $4.7 \pm 0.6 \mathrm{pN}$ (AurB*-KD), $4.8 \pm 0.6 \mathrm{pN}(0.5 \mu \mathrm{M}$ AurB*), or 5.0 $\pm 0.2 \mathrm{pN}\left(5 \mu \mathrm{M}\right.$ Aur $\left.B^{*}\right)$. Error bars represent uncertainty due to counting statistics ( $n=20$ events and 11 detachments (AurB $\left.{ }^{*}-K D\right), n=4$ events and 4 detachments $\left(0.5 \mu \mathrm{M}\right.$ AurB $\left.{ }^{\star}\right)$, or $n=12$ events and 8 detachments $\left(5 \mu M\right.$ AurB $\left.{ }^{\star}\right)$ ). 
bioRxiv preprint doi: https://doi.org/10.1101/415992; this version posted September 13, 2018. The copyright holder for this preprint (which was not certified by peer review) is the author/funder, who has granted bioRxiv a license to display the preprint in perpetuity. It is made availablFigure S1 under aCC-BY-NC-ND 4.0 International license.

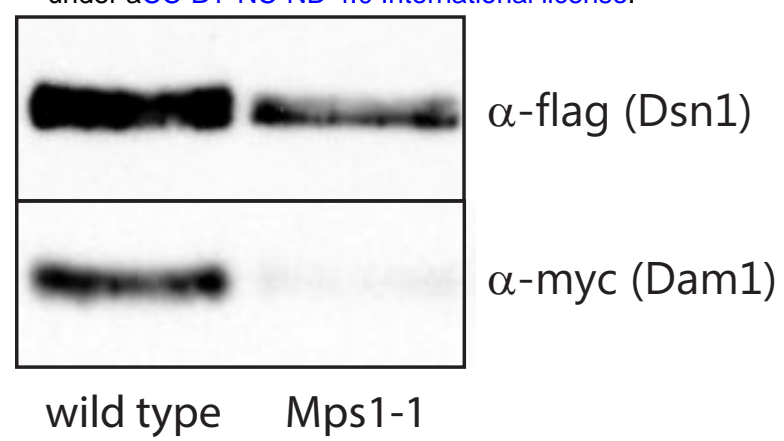

Fig. S1 | Immunoblot of purified kinetochores from either wild-type (SBY8253) or Mps1-1 (SBY8726) kinetochores showing that Mps1-1 kinetochores lack co-purifying Dam1 complex.

\section{a}

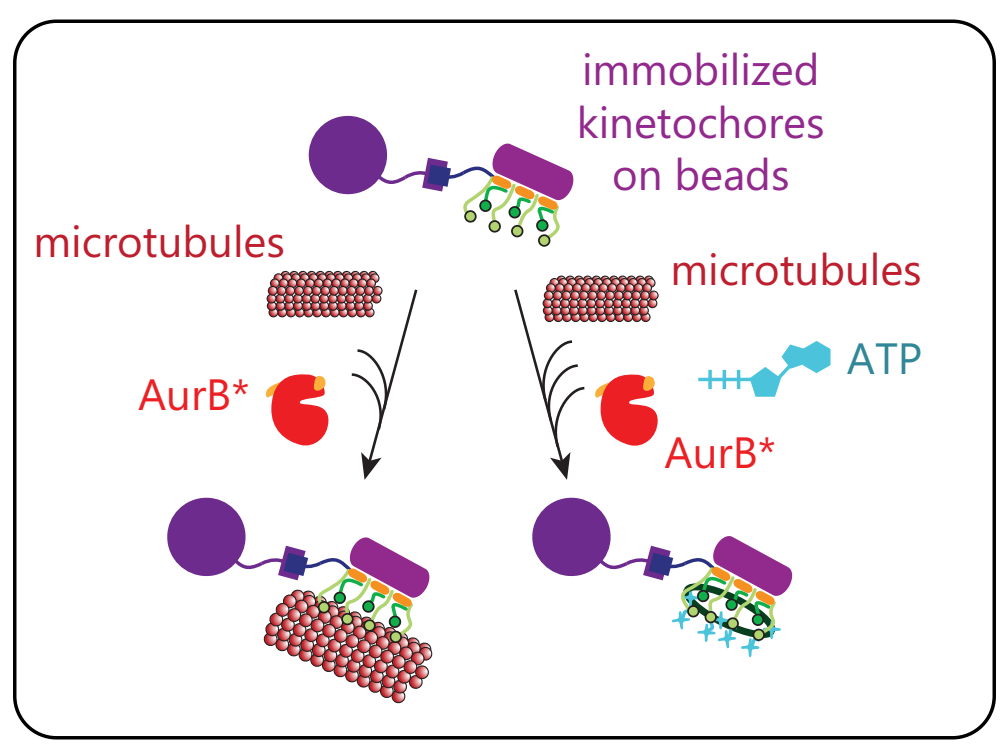

Figure S2

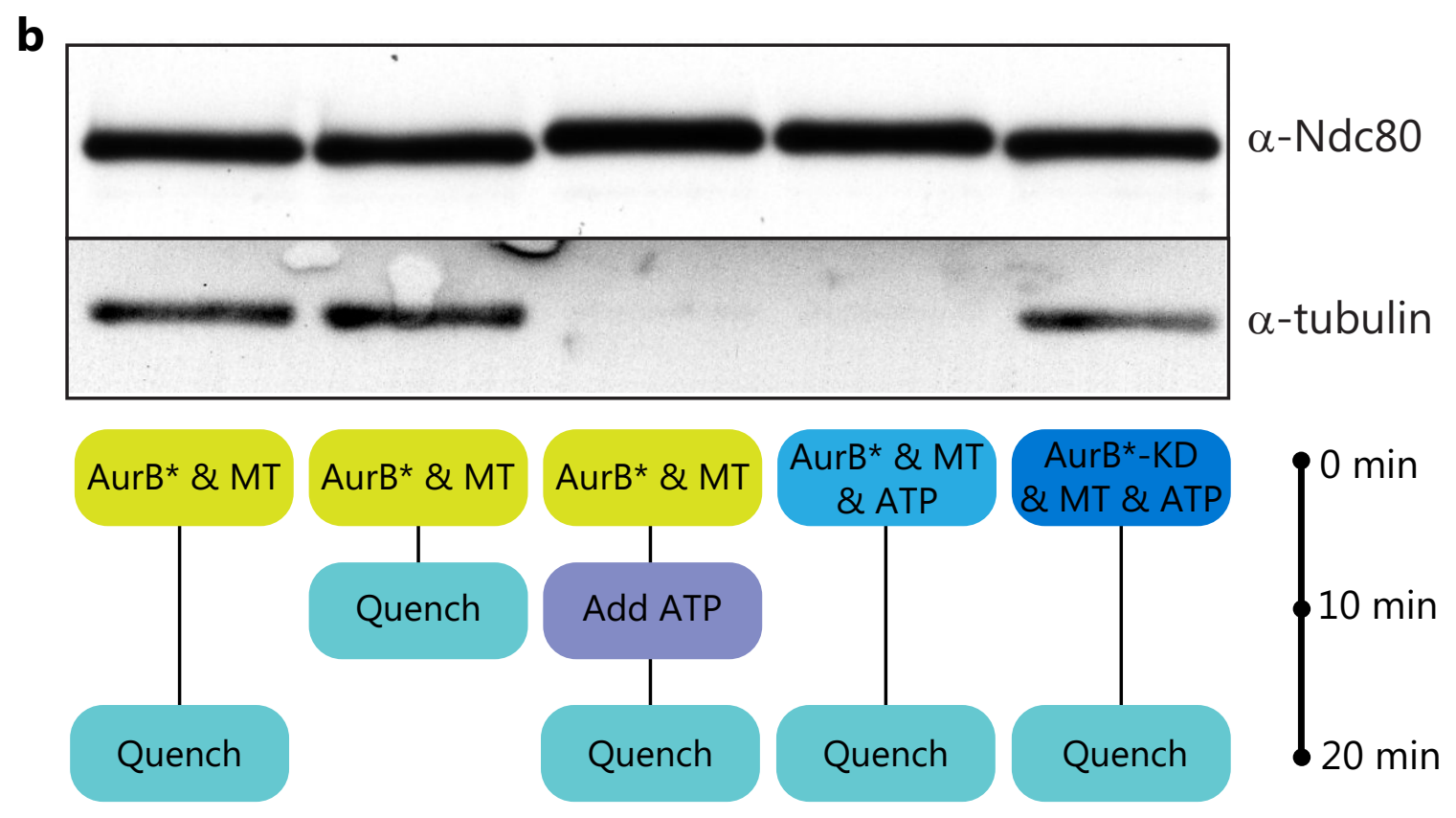

Fig. S2 | a Schematic for microtubule binding experiment b Mps1-1 kinetochores (SBY8726) were incubated with microtubules and kinase for the times indicated in the presence or absence of ATP. Bound components were assayed by Western blot. 

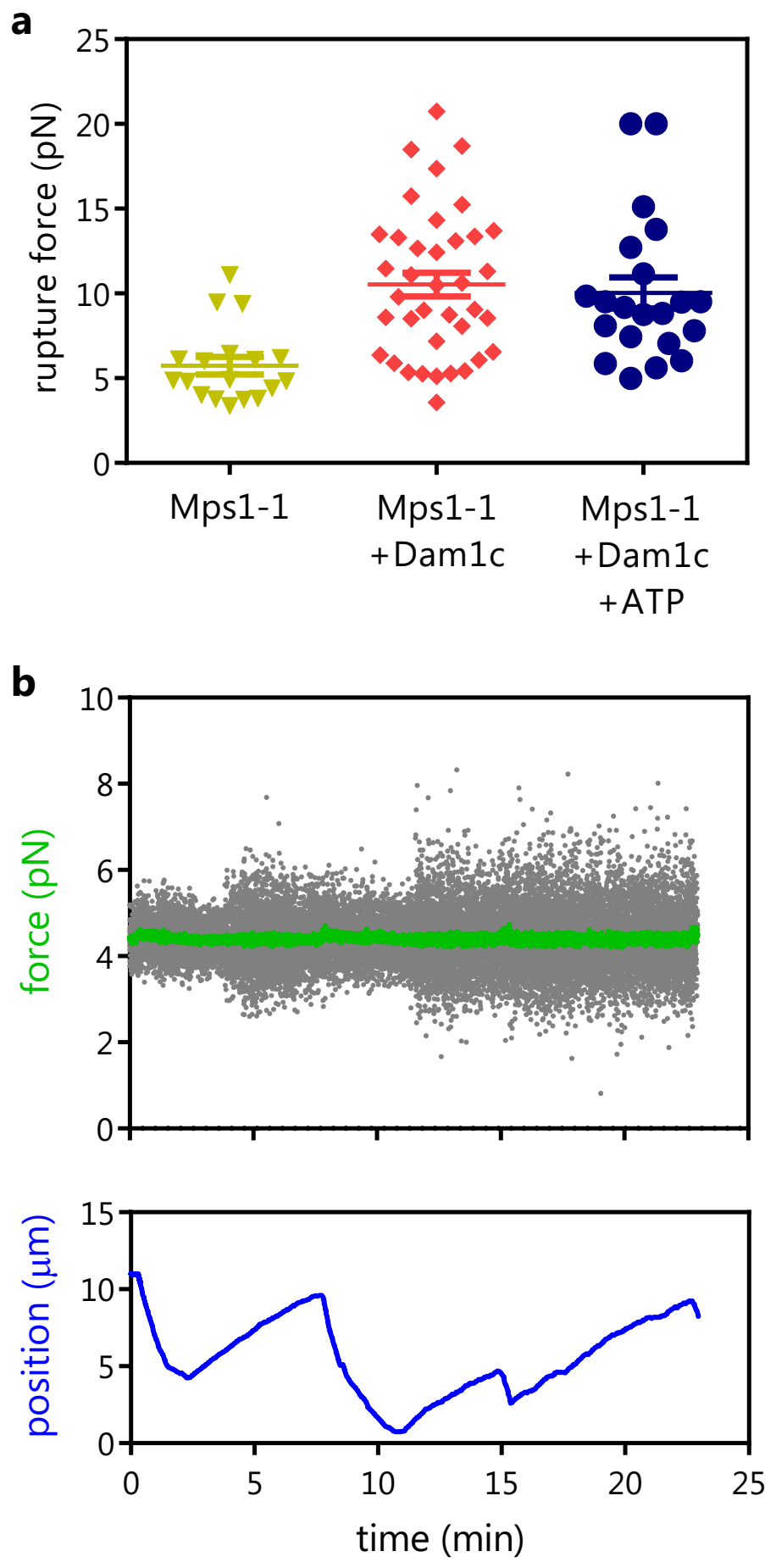

Fig. S3 | a Rupture force values for various kinetochores detaching from microtubules as determined using an optical force-ramp. Where indicated, exogenously purified Dam1 complex (Dam1c) and/or ATP were included in the trapping buffer. $\mathbf{b}$ Traces of optical trap data for an individual force-clamp event. Gray points show the force applied across the kinetochore-microtubule attachment and green points show the same data after smoothing. The blue trace shows the relative position of a single kinetochore-coupled bead as it tracks with the microtubule tip. 

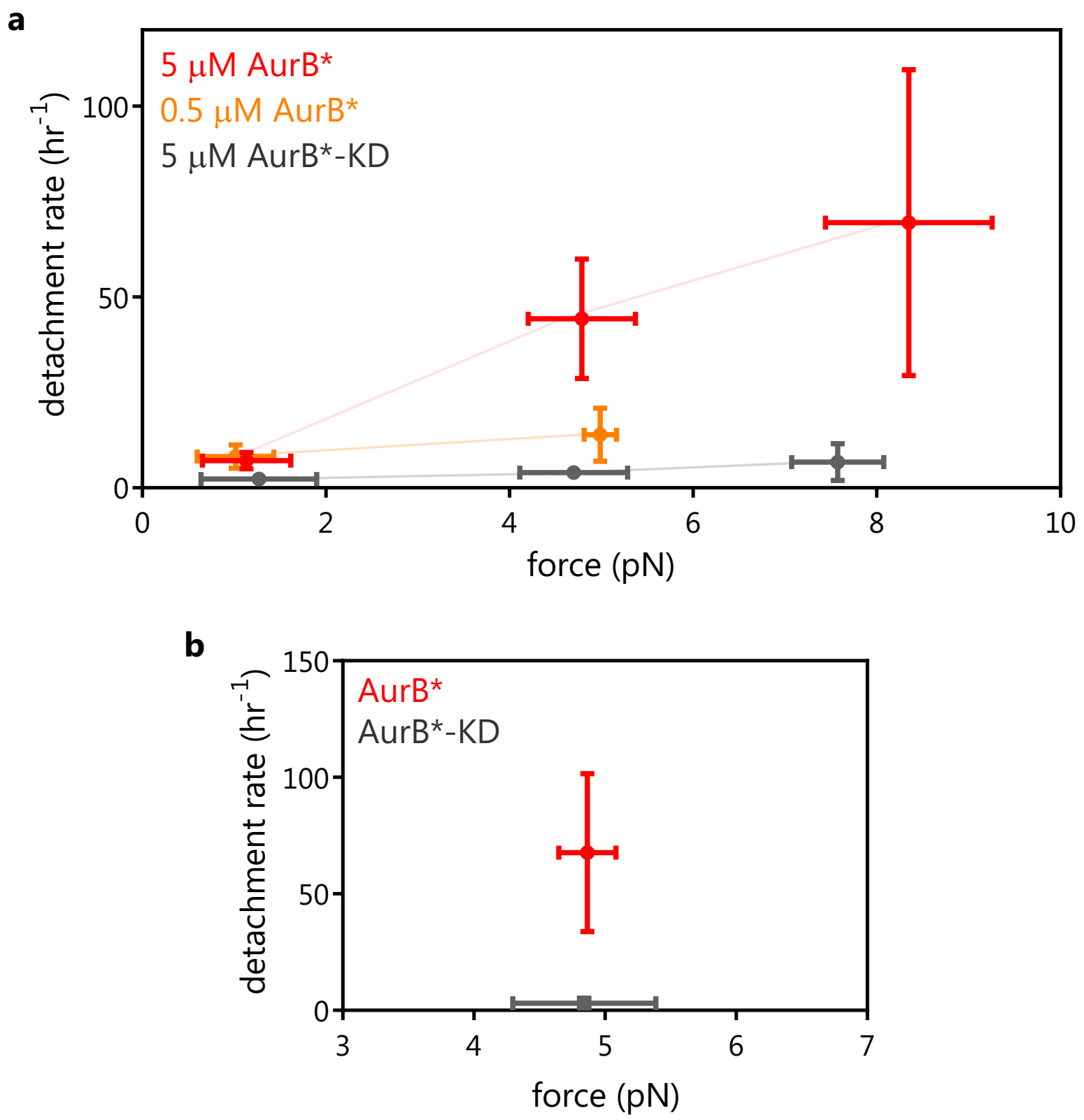

Fig. S4 | a Detachment rates for Mps1-1 kinetochores with Dam1 complex present, separated by concentration of kinase. The data at $1 \mathrm{pN}$ and $5 \mathrm{pN}$ are replotted from Figs. 4 and 5. For the data at $8 \mathrm{pN}, \mathrm{n}=4$ events and 2 detachments (AurB*-KD) or $n=3$ events and 3 detachments $(5 \mu \mathrm{M}$ AurB*). b Detachment rates for wild-type kinetochores at high force. The assay was performed as diagramed in Fig. 3a, except with wild-type kinetochores and no addition of Dam1 complex. $5 \mu \mathrm{M}$ of either AurB* or AurB* ${ }^{\star}$ KD was flowed in. 


\section{Growth}

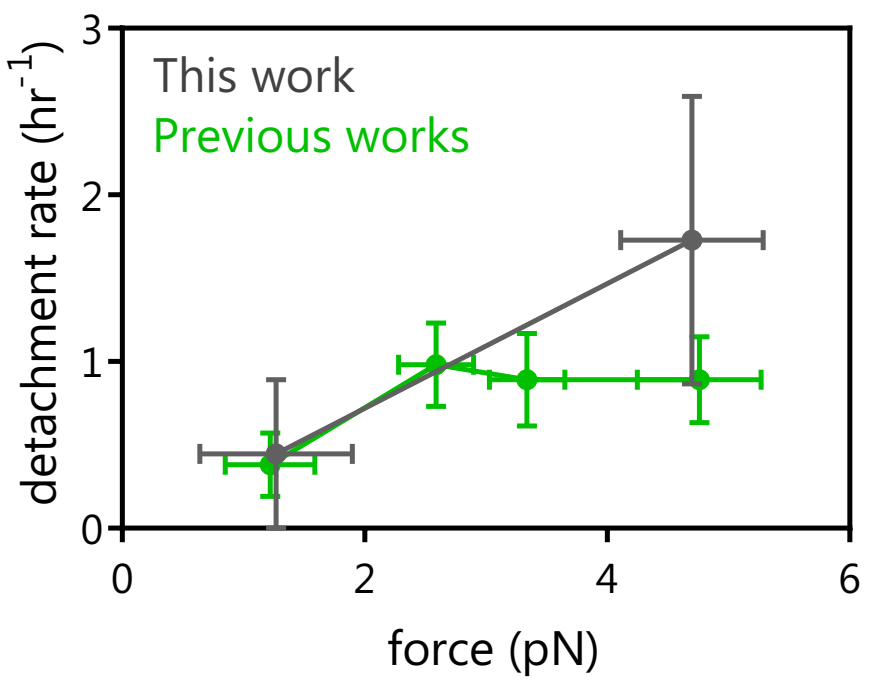

Shortening

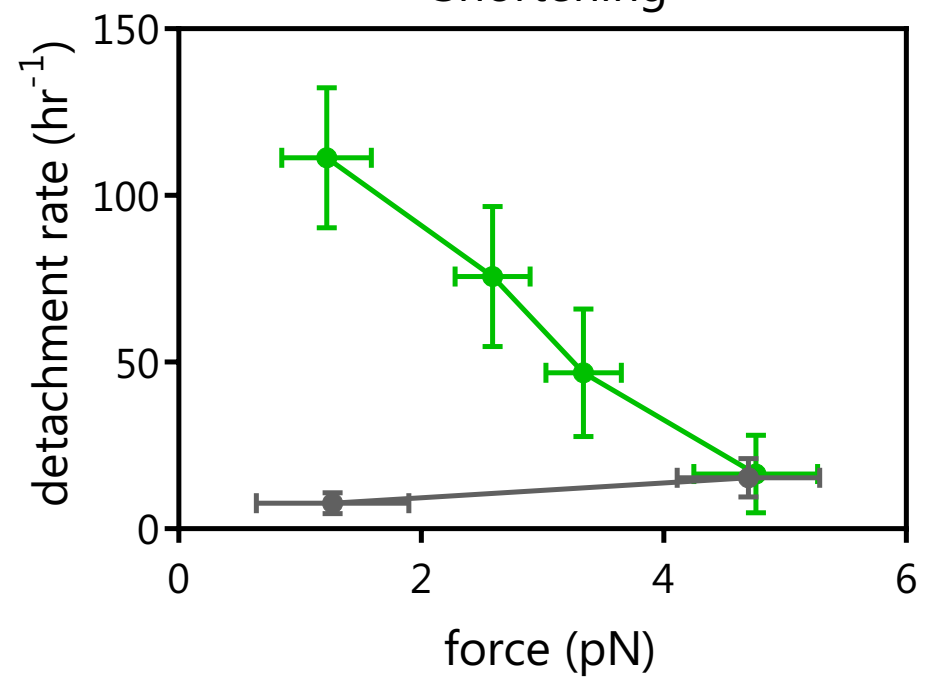

Fig. S5 | a and b Kinetochore detachment rates measured specifically during microtubule growth (a) and during microtubule shortening (b). Rates measured for this work, using Mps1-1 kinetochores with Dam1 complex and AurB*KD flow, are shown in gray. Rates for wild-type kinetochores, from previous work ${ }^{41,53}$, are shown in green. 
Tables

Table 1 | Yeast strains used in this study

SBY8253 MATa ura3-1 leu2,3-112 his3-11 trp1-1 ade2-1 LYS2 can1-100 bar1-1 DSN1-6HISFLAG::URA3

SBY8522 MATa ura3-1 leu2,3-112 his3-11 trp1-1 ade2-1 LYS2 can1-100 bar1-1 DSN1-6HIS3FLAG::URA3 ndc80::NAT::ndc80(T21A,S37A,T54A,T71A,T74A,S95A, S100A)::TRP1

SBY8726 MATa ura3-1 leu2,3-112 his3-11 trp1-1 ade2-1 LYS2 can1-100 bar1-1 DSN1-6His3Flag::URA3 mps1-1

SBY12464 MATa ura3-1 leu2,3-112 his3-11 trp1-1 ade2-1 LYS2 can1-100 bar1-1 DAD13Flag::TRP1

Table 2 | Kinetochore-microtubule detachment rates

\begin{tabular}{|c|c|c|c|c|}
\hline flow with & force $(\mathrm{pN})$ & $\begin{array}{c}\text { error in } \\
\text { force }\end{array}$ & $\begin{array}{l}\text { detachment } \\
\text { rate }\left(\mathrm{min}^{-1}\right)\end{array}$ & $\begin{array}{c}\text { error in } \\
\text { detachment } \\
\text { rate }\end{array}$ \\
\hline \multirow[t]{3}{*}{ AurB*-KD } & 1.27 & 0.63 & 2.31 & 0.87 \\
\hline & 4.70 & 0.59 & 3.97 & 1.20 \\
\hline & 7.57 & 0.50 & 6.76 & 4.78 \\
\hline \multirow[t]{3}{*}{$5 \mu \mathrm{M}$ AurB* } & 1.13 & 0.48 & 7.17 & 2.16 \\
\hline & 4.79 & 0.58 & 44.3 & 15.6 \\
\hline & 8.35 & 0.91 & 69.4 & 40.1 \\
\hline \multirow[t]{2}{*}{$0.5 \mu \mathrm{M}$ AurB* } & 1.02 & 0.42 & 8.24 & 3.11 \\
\hline & 4.99 & 0.18 & 13.9 & 6.97 \\
\hline \multirow[t]{3}{*}{ combined AurB* } & 1.09 & 0.45 & 7.57 & 1.78 \\
\hline & 4.84 & 0.51 & 25.6 & 7.41 \\
\hline & 8.35 & 0.91 & 69.4 & 40.1 \\
\hline
\end{tabular}




\section{Supplementary Note}

Our previous work has shown that tension can directly stabilize reconstituted attachments between kinetochores and dynamic microtubule tips ${ }^{41,53}$, but we did not see this "catch bond-like" effect in the present study. We do not know the precise reasons for the difference, but it probably arose from changes in experimental conditions that were implemented here to enable measurement of Aurora-triggered detachments, as detailed below.

The catch bond-like behavior depends on a combination of two effects, tension-dependent suppression of microtubule shortening, and rapid detachment during shortening ${ }^{41,53}$. In the present study, kinetochore detachment during shortening was relatively rare, occurring at a rate of only $7.7 \pm 3.1 \mathrm{hr}^{-1}$ at $1 \mathrm{pN}$ of tension, whereas previously it was more frequent, occurring at $>100 \mathrm{hr}^{-1}$ at $1 \mathrm{pN}$ (Fig. S5). Thus, the overall release rate in the absence of kinase activity remained relatively low at all forces tested (Figs. 5a, S4a). This behavior fortuitously allowed kinase-triggered release to be detected more readily but eliminated the catch bond-like effect.

Mps1-1 kinetochore particles were used here to avoid the potentially confounding influence of Mps1 kinase, which co-purifies with the wild-type particles used previously. The Mps1-1 kinetochores were bound at a relatively higher density on the beads, to help facilitate microtubule binding during assay setup, and excess free Dam1 was added, to reduce spontaneous detachment. We speculate that one or more of these changes might explain the reduced kinetochore detachment rates during microtubule shortening. 